Few-Body Systems 4, 179-209 (1988)

\title{
Pairing Correlations: II. Exact Model Ground-State Results for Generalised Ladders
}

\author{
R. F. Bishop, W. Piechocki*, and G. A. Stevens \\ Theoretical Physics Group, Department of Mathematics, University of Manchester Institute of \\ Science and Technology, P. O. Box 88, Manchester M60 1QD, England
}

\begin{abstract}
In this series of papers, the so-called ground-state version of the $[\exp (S)$ or] coupled-cluster formalism (CCF) of quantum many-body theory is applied to the general problem of pairing correlations within a many-body system of identical fermions. In this second work in the series we restrict ourselves to exact calculations and concentrate on analytic solutions to the generalised ladder approximations formulated in the first paper. We focus attention on the particular model case of a general (non-local) separable potential, and work within the so-called complete ladder (CLAD) approximation which was shown in the earlier paper to be the CCF formulation of the well-known Galitskii approximation. We show how the CLAD approximation reduces in this case to a highly non-trivial pair of coupled nonlinear integral equations for the four-point correlation function, $S_{2}$, which provides a measure of the two-particle/two-hole component in the true "ground-state" wavefunction. In the further derivation of exact analytic solutions for both $S_{2}$ and the corresponding "ground-state" energy, we also see how various types of composite pairs within the many-body medium manifest themselves as "virtual (de-)excitations". We thus show how our CCF provides an efficient and unified framework in which to describe all aspects of pairing, such as: (i) a possible free bound pair and its gradual approach to "dissolution" as the density is increased; (ii) the possible appearance of a second bound pair of predominantly hole-like quasi-particles above some lower critical density (which depends on the total momentum of the pair); (iii) the unstable but bound resonant pairs that can exist for densities above a comparable upper critical density at which the two previous types of real bound pairs have "dissolved"; and (iv) Cooper pairs. Even though each of these composite pairs leads to a new "condensed-pair phase" of lower energy, we further show that our so-called ground-state CCF leads only to the fluid-like state of uncondensed particles. In a third paper in this series we use the solutions obtained here as input to the analogous excited-state version of the $\mathrm{CCF}$, and show how these various composite pairs materialise as "negative energy (de-)excitations".
\end{abstract}

* On leave of absence from: Nuclear Theory Department, Institute for Nuclear Studies, Hoża 69, PL-00-681 Warsaw, Poland 


\section{Introduction}

The present paper is the second in a series which considers the general problem of pairing correlations within a many-body system of identical fermions. In the previous first paper [1] (hereafter referred to as I), to which the reader is directed, we gave a rather full motivation for and historical discussion of this problem. We demonstrated furthermore how the ground-state (g.s.) version of the so-called $[\exp (S)$ or $]$ coupled-cluster formalism $(\mathrm{CCF})$ of quantum many-body theory may be rather generally applied to it. It was shown in I how the CCF provides a particularly powerful and unifying approach to the very broad problem of investigating such sub-structure as bound clusters within an interacting many-body system.

Particular attention was paid in I to the pairing phenomena that arise due to particle-particle (pp) and hole-hole (hh) correlations, and it was shown how the CCF could be used to express the various generalised ladder approximations that arise from correlations in these two ( $\mathrm{pp}$ and $\mathrm{hh}$ ) channels, treated simultaneously. We specifically demonstrated how one could formulate within the CCF each of: (i) the Bethe-Goldstone (BG) approximation [2] which keeps only the pp-ladders (PPLAD); (ii) an intermediate approximation that adds to PPLAD the hh-ladders (HHLAD), and which seems to have been investigated earliest by Chisholm and Squires [3] and by Mehta [4]; and (iii) the so-called complete ladder (CLAD) approximation that adds to PPLAD and HHLAD the mixed pp/hh-ladders (MLAD). This latter approximation is fully equivalent to the so-called Galitskii approximation within the Galitskii-Feynman (GF) formalism, which is itself just the ladder approximation to the Bethe-Salpeter equation [5].

The lowest level of truncation of the CCF which goes beyond an independentparticle (Hartree-Fock) approach is the so-called SUB2 approximation. We pointed out in I how this SUB2 approximation fully describes the most general aspects of pairing within a many-body system. In particular, it couples together simultaneously, and treats on an equal footing, the $\mathrm{pp}$, hh, and particle-hole (ph) channels. We saw explicitly how the CCF may be cast, at this SUB2 level, as a nonlinear integral equation for a four-point correlation function, $S_{2}$, which describes the two-particle/two-hole component of the exact g.s. wavefunction $|\Psi\rangle$ of the interacting system.

In many ways the present paper and its predecessor [1] may be viewed as the analogue for pp-hh channels of an earlier paper [6] by one of the current authors and his co-workers, which dealt with the correlations in the ph channel as a precursor to a full calculation [7] of electron correlations within the one-component Coulomb system (or "electron gas"). In that particular paper [6], we focussed attention in the first instance on the random-phase approximation (RPA) [8] within the CCF. We showed that the RPA could be expressed within the CCF at the SUB2 level, and in terms of a highly non-trivial, nonlinear (- actually bilinear) integral equation for the four-point function $S_{2}$. In particular, we found the exact, analytic solutions of this equation in the case of local pairwise potential interactions between the particles. It is our main aim again in the present work to restrict ourselves to exact calculations within the pp-hh channels, and to concentrate on analytic solutions to the generalised ladder approximations, particularly the most general CLAD approximation, formulated in I. To this end we restrict ourselves here to the 
particular model case where the particles interact via non-local pairwise potentials of the separable kind.

We find that both the RPA equation and the CLAD equation for $S_{2}$, each of which arises as a distinct sub-approximation to the full SUB2 approximation, are bilinear integral equations. In the present paper, we draw on our earlier experience of the RPA in the ph channels with local potentials, to help solve the corresponding CLAD approximation in the pp-hh channels with separable potentials. We note that separable potentials are chosen here purely so as to be able to obtain analytic solutions. However, we should point out that there is no particular problem with solving the CLAD equations numerically for local potentials. Moreover, even rather general local potentials may be very well approximated by choosing a sum of a sufficiently large number of suitable separable terms.

The interested reader is referred back to I for further introductory remarks and a fuller discussion of the CCF. In Sect. 2 we present a brief resume of the formalism and the imbedding within it of the generalised ladder approximations with which we are mostly concerned here. In Sect. 3 we then solve, in the case of separable potentials, for the two-body correlation function $S_{2}$, for both the CLAD and PPLAD cases that correspond respectively to the Galitskii and BG approximations. Exact analytic expressions are given in each case. The results are discussed and compared in Sect.4, with special attention being given to the various types of composite-pair states that emerge from these respective calculations in the coupled pp-hh channels and pp channel only. Further extensions of the work are also discussed in Sect. 4.

\section{Ladder Approximations Within the CCF}

We consider a homogeneous system of $N$ identical spin- $\frac{1}{2}$ particles, of mass $m$ each, contained in a normalisation box of volume $\Omega$, and interacting via two-body potentials. We shall ultimately be interested in the thermodynamic limit where $N \rightarrow \infty, \Omega \rightarrow \infty$, such that the density $\rho=N / \Omega$ remains finite. It is thus convenient to choose to work in a plane-wave single-particle (s.p.) basis of states, $|\vec{k} s\rangle$, labelled by wavenumber $\vec{k}$ and spin-projection $s$ along some fixed quantisation axis. Very specifically, these s.p. states are given in the coordinate-space representation as

$$
\langle\vec{r} \mid \vec{k} s\rangle=\Omega^{-1 / 2} \exp (i \vec{k} \cdot \vec{r}) \eta(s),
$$

with $\eta(s)$ the two-component spin wavefunction. If we denote s.p. creation and destruction operators as the Hermitian conjugates $a_{\vec{k} s}^{\vec{\uparrow}}$ and $a_{\vec{k} s}$ respectively, we have

$$
|\vec{k} s\rangle=a_{k s}^{\dagger}|0\rangle,
$$

where $|0\rangle$ is the vacuum state. Uncorrelated but antisymmetrised $n$-body states are then represented as

$$
\left|\vec{k}_{1} s_{1} ; \cdots ; \vec{k}_{n} s_{n}\right\rangle_{A} \equiv a_{\vec{k}_{1} 1}^{\dagger} \cdots a_{\vec{k}_{n} s_{n}}^{\dagger}|0\rangle
$$

Alternatively, the uncorrelated but non-antisymmetrised $n$-body states may be represented as the direct product states

$$
\left|\vec{k}_{1} s_{1} ; \cdots ; \vec{k}_{n} s_{n}\right\rangle \equiv\left|\vec{k}_{1} s_{1}\right\rangle \cdots\left|\vec{k}_{n} s_{n}\right\rangle
$$


Henceforth we shall deal only with systems interacting via spin-independent pairwise potentials, $V$. Taking Galilean invariance into account explicitly, we have, in terms of relative and centre-of-mass momentum variables,

$\left\langle\frac{1}{2} \vec{P}+\vec{p}, s_{1} ; \frac{1}{2} \vec{P}-\vec{p}, s_{2}|V| \frac{1}{2} \vec{P}+\vec{p}^{\prime}, s_{3} ; \frac{1}{2} \vec{P}-\vec{p}^{\prime}, s_{4}\right\rangle=\left\langle\vec{p}|V| \vec{p}^{\prime}\right\rangle \delta_{s_{1} s_{3}} \delta_{s_{2} s_{4}}$,

independent of the total momentum $\vec{P}$ of the interacting pair.

We have seen in I how, in terms of the usual uncorrelated, antisymmetrised product of the lowest $N$ s.p. states (of the form of Eq. (2.3) with the $N$ wavenumbers chosen to be the lowest eigenstates in the normalisation box up to a Fermi wavenumber $k_{F}$ ), which forms the usual zeroth-order Slater determinant reference state $|\Phi\rangle$, the $C C F$ represents the exact ground-state (g.s.) wavefunction $|\Psi\rangle$ of the interacting system in the lowest SUB2 level of approximation as

$$
\begin{gathered}
|\Psi\rangle=\exp \left(S_{2}\right)|\Phi\rangle, \\
S_{2}=\frac{1}{(2 !)^{2}} \sum_{s_{1}, s_{2}} \sum_{\vec{p}, \vec{p}^{\prime}, \vec{P}} S_{2 ; \vec{p} \vec{p}^{\prime}}^{s_{1} s_{2}}(\vec{P}) a_{\frac{1}{2} \vec{p}+\vec{p}, s_{1}}^{\dagger} a_{\frac{1}{2} \vec{p}+\vec{p}, s_{2}}^{\dagger} a_{\frac{1}{2} \vec{P}-\vec{p}^{\prime}, s_{2}} a_{\frac{1}{2} \vec{P}+\vec{p}^{\prime}, s_{1}} .
\end{gathered}
$$

We have discussed in detail in I how the wavefunction of Eq. (2.6) couples the particle-particle, hole-hole and particle-hole correlations simultaneously and on an equal footing. The derivation of the SUB2 equation for the matrix elements of the operator $S_{2}$ expressed in Eq. (2.7), is described in detail in I. The antisymmetrised nature of the matrix elements $S_{2 ; \vec{p} \vec{p}^{\prime}}^{s_{1} s_{2}}(\vec{P})$ in Eq. (2.7) can be made explicit, for a system whose exact g.s. is assumed to share the invariance under spatial reflections of the underlying Hamiltonian, as

$$
S_{2 ; \vec{p} \vec{p}^{\prime}}^{s_{1} s_{2}}(\vec{P})=T_{\vec{p} \vec{p}^{\prime}}(\vec{P})-\delta_{s_{1} s_{2}} T_{\vec{p},-\vec{p}^{\prime}}(\vec{P}),
$$

with the non-antisymmetrised matrix element $T_{\vec{p} \vec{p}^{\prime}}(\vec{P})$ given by the expression

$$
T_{\vec{p} \vec{p}^{\prime}}(\vec{P})=\left\langle\frac{1}{2} \vec{P}+\vec{p}, s ; \frac{1}{2} \vec{P}-\vec{p}, s\left|S_{2}\right| \frac{1}{2} \vec{P}+\vec{p}^{\prime}, s ; \frac{1}{2} \vec{P}-\vec{p}^{\prime}, s\right\rangle,
$$

independent of the spin projection $s$.

We have explained rather fully in I how the complete ladder (CLAD) approximation is that further approximation (-fully equivalent to the GalitskiiFeynman approximation -) to the CCF SUB2 approximation, which incorporates all of the (mixed) particle-particle and hole-hole ladder terms simultaneously, but which ignores all particle-hole correlations (and self-energy insertions). The CLAD approximation is expressed graphically by Fig. 1 of I, and algebraically by the nonlinear equation (2.21) of $\mathrm{I}$, which we reproduce here,

$$
\begin{aligned}
& \frac{\hbar^{2}}{m}\left(p^{2}-p^{\prime 2}\right) T_{\vec{p} \vec{p}^{\prime}}(\vec{P})+\left\langle\vec{p}|V| \vec{p}^{\prime}\right\rangle \\
& \quad+\sum_{\vec{k}}\langle\vec{p}|V| \vec{k}\rangle T_{\vec{k}^{p^{\prime}}}(\vec{P}) \bar{Q}(\vec{k}, \vec{P})+\sum_{\vec{k}^{\prime}} T_{\vec{p} \vec{p}^{\prime}}(\vec{P})\left\langle\vec{k}^{\prime}|V| \vec{p}^{\prime}\right\rangle Q\left(\vec{k}^{\prime}, \vec{P}\right) \\
& \quad+\sum_{\vec{k}, \vec{k}^{\prime}} T_{\vec{p} \vec{p}^{\prime}}(\vec{P})\left\langle\vec{k}^{\prime}|V| \vec{k}\right\rangle T_{\vec{k}^{\prime}}, \bar{Q}(\vec{k}, \vec{P}) Q\left(\vec{k}^{\prime}, \vec{P}\right)=0 .
\end{aligned}
$$

The factors $Q\left(\vec{p}^{\prime}, \vec{P}\right)$ and $\bar{Q}(\vec{p}, \vec{P})$ in Eq. (2.10) respectively restrict the associated sums to run over pairs of momenta associated with the intermediate scattering states of two holes inside the Fermi sea and two particles outside the Fermi sea, 
respectively. Thus,

$Q\left(\vec{p}^{\prime}, \vec{P}\right) \equiv n\left(\frac{1}{2} \vec{P}+\vec{p}^{\prime}\right) n\left(\frac{1}{2} \vec{P}-\vec{p}^{\prime}\right), \quad \bar{Q}(\vec{p}, \vec{P})=\bar{n}\left(\frac{1}{2} \vec{P}+\vec{p}\right) \bar{n}\left(\frac{1}{2} \vec{P}-\vec{p}\right)$,

in terms of the unperturbed hole and particle distributions, $n(k)$ and $\bar{n}(k)$,

$$
n(k) \equiv \theta\left(k_{F}-k\right), \quad \bar{n}(k) \equiv \theta\left(k-k_{F}\right),
$$

where $\theta(x)$ is the usual unit-step function, defined to be one (zero) for $x$ greater (less) than zero.

We have also derived the g.s. energy, $E$, of the system in the SUB2 approximation as Eq. (2.23) of I, which we again reproduce as,

$$
\begin{aligned}
E= & 2 \sum_{\vec{k}} \frac{\hbar^{2} k^{2}}{2 m} n(k)+\sum_{\vec{p}^{\prime}, \vec{P}} Q\left(\vec{p}^{\prime}, \vec{P}\right)\left[2\left\langle\vec{p}^{\prime}|V| \vec{p}^{\prime}\right\rangle-\left\langle\vec{p}^{\prime}|V|-\vec{p}^{\prime}\right\rangle\right. \\
& \left.+\sum_{\dot{p}}\left\langle\vec{p}^{\prime}|V| \vec{p}\right\rangle\left\{2 T_{\vec{p} \vec{p}^{\prime}}(\vec{P})-T_{\vec{p},-\vec{p}^{\prime}}(\vec{P})\right\} \bar{Q}(\vec{p}, \vec{P})\right],
\end{aligned}
$$

where the factors of 2 are from the spin sums.

Finally, in the thermodynamic limit in which we are interested, the s.p. sums are replaced by integrals in the usual way,

$$
\sum_{\vec{k}} f(\vec{k}) \rightarrow \Omega(2 \pi)^{-3} \int d \vec{k} f(\vec{k})
$$

for an arbitrary function $f(\vec{k})$. In particular, we have as usual that for a spinsaturated spin- $\frac{1}{2}$ system, the Fermi wavenumber $k_{F}$ is given by $k_{F}=\left(3 \pi^{2} \rho\right)^{1 / 3}$.

In the following section, we shall show how the nonlinear CLAD integral equation (2.10) may be solved, exactly and analytically, in the particular case of a non-local model potential of the separable kind.

\section{Application to Separable Potentials}

\subsection{General Comments}

The most general rotationally-invariant separable potential can be written in the operator form

$$
V=\sum_{l=0}^{\infty} \sum_{n=1}^{N_{l}} C_{n l}\left|g_{n l}\right\rangle\left\langle g_{n l}\left|\sum_{m}\right| l m\right\rangle\langle l m|,
$$

where the states $|l m\rangle$ are the usual spherical harmonics, and in each partial wave $l$ we consider a rank- $N_{l}$ separable form. The constants $C_{n l}$ are real but may be of either sign. We shall restrict the present discussion to a one-term $S$-wave separable form for simplicity, although the method can also readily be extended to the more general form. In this case we have

$$
\left\langle\vec{r}|V| \vec{r}^{\prime}\right\rangle=\lambda \frac{\hbar^{2}}{m} g(r) g^{*}\left(r^{\prime}\right)
$$

where $\langle r \mid g\rangle \equiv g(r), \lambda$ is the strength of the interaction, and the factor $\hbar^{2} m^{-1}$ has been separated for later convenience. Equivalently, in our plane-wave (momentumspace) basis, we have 


$$
\begin{aligned}
\left\langle\vec{p}|V| \vec{p}^{\prime}\right\rangle & =\lambda \frac{\hbar^{2}}{m} \frac{1}{\Omega} g(p) g^{*}\left(p^{\prime}\right), \\
g(p) & =\int d \vec{r} e^{-i \vec{p} \cdot \vec{r}} g(r) .
\end{aligned}
$$

We note that if $\lambda$ is considered dimensionless, then $g(r)$ has dimensions $L^{-5 / 2}$ and $g(p)$ has dimensions $L^{1 / 2}$ where $L$ is a length.

\subsection{The CLAD (GF) Approximation}

As already discussed the Galitskii-Feynman (GF) approximation is now analogous in our CCF to solving the CLAD equation (2.10), for the matrix elements $T_{\vec{p} \vec{p}^{\prime}}(\vec{P})$. Inserting the separable potential (3.3) into Eq. (2.10) and passing to the thermodynamic limit as in Eq. (2.14), yields the factorizable form

$$
\begin{aligned}
\left(p^{2}-p^{\prime 2}\right) T_{\vec{p} \vec{p}^{\prime}}(\vec{P})= & -\frac{\lambda}{\Omega}\left[g(p)+\Omega \int \frac{d \vec{k}^{\prime}}{(2 \pi)^{3}} g\left(k^{\prime}\right) T_{\vec{p} \overrightarrow{k^{\prime}}}(\vec{P}) Q\left(\vec{k}^{\prime}, \vec{P}^{\prime}\right)\right] \\
& \times\left[g^{*}\left(p^{\prime}\right)+\Omega \int \frac{d \vec{k}}{(2 \pi)^{3}} g^{*}(k) T_{\vec{k} \vec{p}^{\prime}}(\vec{P}) \bar{Q}(\vec{k}, \vec{P})\right] .
\end{aligned}
$$

Equation (3.4) can thus be written in the form

$$
T_{\tilde{p} \dot{p}^{\prime}}(\vec{P})=-\frac{\lambda}{\Omega} \frac{g(p) g^{*}\left(p^{\prime}\right)}{p^{2}-p^{\prime 2}} l\left(p^{2}\right) b\left(p^{\prime 2}\right),
$$

where the functions $l\left(p^{2}\right)$ and $b^{*}\left(p^{\prime 2}\right)$ are themselves given in terms of $T$ by

$$
\begin{gathered}
l(\vec{p}, \vec{P}) \equiv l\left(p^{2}\right)=1+\frac{1}{g(p)} \Omega \int \frac{d \vec{k}^{\prime}}{(2 \pi)^{3}} g\left(k^{\prime}\right) T_{\vec{p} \vec{k}^{\prime}}(\vec{P}) Q\left(\vec{k}^{\prime}, \vec{P}^{\prime}\right), \\
b\left(\vec{p}^{\prime}, \vec{P}\right) \equiv b\left(p^{\prime 2}\right)=1+\frac{1}{g^{*}\left(p^{\prime}\right)} \Omega \int \frac{d \vec{k}}{(2 \pi)^{3}} g^{*}(k) T_{\vec{k}^{\prime}}(\vec{P}) \bar{Q}(\vec{k}, \vec{P}) .
\end{gathered}
$$

It should be noted that the functions $l\left(p^{2}\right)$ and $b\left(p^{\prime 2}\right)$, also both depend on the total momentum $\vec{P}$, but for ease of notation we henceforth suppress this dependence, as indicated in Eq. (3.6). Inserting the form (3.5) into Eqs. (3.6) then shows that the CLAD (or GF) approximation within the CCF has reduced to solving the following coupled pair of nonlinear integral equations for the functions $l\left(p^{2}\right)$ and $b\left(p^{\prime 2}\right)$,

$$
\begin{gathered}
l\left(p^{2}\right)=1-\lambda l\left(p^{2}\right) \int_{0}^{\infty} \frac{k^{\prime 2} d k^{\prime}}{2 \pi^{2}} \frac{\left|g\left(k^{\prime}\right)\right|^{2}}{p^{2}-k^{\prime 2}} b\left(k^{\prime 2}\right) q\left(k^{\prime}, P\right), \\
b\left(p^{\prime 2}\right)=1-\lambda b\left(p^{\prime 2}\right) \int_{0}^{\infty} \frac{k^{2} d k}{2 \pi^{2}} \frac{|g(k)|^{2}}{k^{2}-p^{\prime 2}} l\left(k^{2}\right) \bar{q}(k, P),
\end{gathered}
$$

where the functions $q$ and $\bar{q}$ are the angle-averaged $Q$ and $\bar{Q}$ functions respectively that limit the two-hole intermediate scattering states to be inside the Fermi sea and the two-particle intermediate scattering states to be outside the Fermi sea respectively, 


$$
\begin{aligned}
q\left(k^{\prime}, P\right) & \equiv \frac{1}{4 \pi} \int d \Omega Q\left(\vec{k}^{\prime}, \vec{P}\right) \\
\bar{q}(k, P) & \equiv \frac{1}{4 \pi} \int d \Omega \bar{Q}(\vec{k}, \vec{P})
\end{aligned}
$$

where the integration is over the full angular range $\Omega$ of the directions of $\vec{P}$. Using the explicit forms (2.11) and (2.12), the angular integrations may be performed explicitly in Eq. (3.8) to give

$$
\begin{aligned}
& \bar{q}(k, P)= \begin{cases}0, & k \leqslant\left(k_{F}^{2}-\frac{1}{4} P^{2}\right)^{1 / 2}, \\
\left(k^{2}+\frac{1}{4} P^{2}-k_{F}^{2}\right) / k P, & \left(k_{F}^{2}-\frac{1}{4} P^{2}\right)^{1 / 2}<k<\frac{1}{2} P+k_{F}, \\
1, & k \geqslant \frac{1}{2} P+k_{F},\end{cases} \\
& q\left(k^{\prime}, P\right)= \begin{cases}1, & k^{\prime} \leqslant k_{F}-\frac{1}{2} P, \\
\left(k_{F}^{2}-\frac{1}{4} P^{2}-k^{\prime 2}\right) / k P, & k_{F}-\frac{1}{2} P<k^{\prime}<\left(k_{F}^{2}-\frac{1}{4} P^{2}\right)^{1 / 2}, \\
0, & k^{\prime} \geqslant\left(k_{F}^{2}-\frac{1}{4} P^{2}\right)^{1 / 2},\end{cases}
\end{aligned}
$$

in the case $P \leqslant 2 k_{F}$; and

$$
\begin{array}{ll}
\bar{q}(k, P) & = \begin{cases}1, & k \leqslant \frac{1}{2} P-k_{F}, \\
\left(k^{2}+\frac{1}{4} P^{2}-k_{F}^{2}\right) / k P, & \frac{1}{2} P-k_{F}<k<\frac{1}{2} P+k_{F}, \\
1, & k \geqslant \frac{1}{2} P+k_{F},\end{cases} \\
q\left(k^{\prime}, P\right)=0, &
\end{array}
$$

in the case $P>2 k_{F}$.

Before we turn to a solution of Eqs. (3.7) let us first evaluate the g.s. energy in this case. Inserting the explicit solution (3.5) into Eq. (2.13) gives

$$
\begin{aligned}
E= & \frac{3}{5} \frac{\hbar^{2} k_{F}^{2}}{2 m} N+\Omega \lambda \frac{\hbar^{2}}{m} \int \frac{p^{\prime 2} d p^{\prime}}{2 \pi^{2}} \int \frac{P^{2} d P}{2 \pi^{2}} q\left(p^{\prime}, P\right)\left|g\left(p^{\prime}\right)\right|^{2} \\
& \times\left[1-\lambda b\left(p^{\prime 2}\right) \int \frac{p^{2} d p}{2 \pi^{2}} \frac{|g(p)|^{2}}{p^{2}-p^{\prime 2}} l\left(p^{2}\right) \bar{q}(p, P)\right] .
\end{aligned}
$$

Using Eq. ( 3.7 a), we may finally express the g.s. energy per particle in the form

$$
\frac{E}{N}=\frac{3}{5} \frac{\hbar^{2} k_{F}^{2}}{2 m}+\frac{3}{2} \lambda \frac{\hbar^{2}}{m k_{F}^{3}} \int_{0}^{2 k_{F}} P^{2} d P \int \frac{p^{\prime 2} d p^{\prime}}{2 \pi^{2}}\left|g\left(p^{\prime}\right)\right|^{2} b\left(p^{\prime 2}\right) q\left(p^{\prime}, P\right)
$$

As they stand, the coupled pair of nonlinear equations (3.7) are non-singular in the physical regime (or scattering regime) which is defined, via the defining Eq. (2.11), to be the region where $\left|\frac{1}{2} \vec{P} \pm \vec{p}\right|>k_{F}$ and $\left|\frac{1}{2} \vec{P} \pm \vec{p}^{\prime}\right|<k_{F}$. In this case the energy denominators in Eqs. (3.7) never vanish in the physical regime due to the restrictions implied by the $q$ and $\bar{q}$ functions in the integrands. However, in order to make further progress with the solution of Eqs. (3.7) it appears inevitable to make some appeal to analytic continuation. We therefore define enlarged functions $l(z)$ 
and $b(z)$ over the entire complex energy plane as the solutions to the coupled equations arrived at by analytically continuing Eqs. (3.7),

$$
\begin{gathered}
\lambda \int_{0}^{\infty} \frac{k^{\prime 2} d k^{\prime}}{2 \pi^{2}} \frac{\left|g\left(k^{\prime}\right)\right|^{2}}{z-k^{\prime 2}} b\left(k^{\prime 2}\right) q\left(k^{\prime}, P\right)=\frac{1}{l(z)}-1, \\
\lambda \int_{0}^{\infty} \frac{k^{2} d k}{2 \pi^{2}} \frac{|g(k)|^{2}}{k^{2}-z} l\left(k^{2}\right) \bar{q}(k, P)=\frac{1}{b(z)}-1 .
\end{gathered}
$$

Although our basic Eqs. (3.11) are two coupled equations, nevertheless their basic structure is remarkably similar to the analogous RPA equation for local potentials in the CCF, given by Eq. (3.26) of ref. [6]. We therefore follow a similar procedure as in ref. [6] to attempt to bring them into linear form. Let us therefore multiply Eq. (3.7a) by the quantity $\lambda p^{2}\left(2 \pi^{2}\right)^{-1}|g(p)|^{2}\left(p^{2}-z\right)^{-1} \bar{q}(p, P)$ and integrate on the variable $p$. (It is possible to perform the same analysis on Eq. (3.7 b) to arrive at the same result.) After a simple algebraic manipulation on the second term on the right-hand side employing the trivial identity,

$$
\frac{1}{\left(p^{2}-z\right)\left(p^{2}-k^{\prime 2}\right)}=\frac{1}{\left(z-k^{\prime 2}\right)}\left[\frac{1}{p^{2}-z}-\frac{1}{p^{2}-k^{\prime 2}}\right]
$$

we find

$$
\begin{aligned}
\lambda \int \frac{p^{2} d p}{2 \pi^{2}} \frac{|g(p)|^{2}}{p^{2}-z} \bar{q}(p, P)= & \lambda \int \frac{p^{2} d p}{2 \pi^{2}} \frac{|g(p)|^{2}}{p^{2}-z} l\left(p^{2}\right) \bar{q}(p, P) \\
& +\lambda^{2} \int \frac{k^{\prime 2} d k^{\prime}}{2 \pi^{2}} \frac{\left|g\left(k^{\prime}\right)\right|^{2}}{z-k^{\prime 2}} b\left(k^{\prime 2}\right) q\left(k^{\prime}, P\right) \\
& \times \int \frac{p^{2} d p}{2 \pi^{2}}|g(p)|^{2}\left(\frac{1}{p^{2}-z}-\frac{1}{p^{2}-k^{\prime 2}}\right) l\left(p^{2}\right) \bar{q}(p, P)
\end{aligned}
$$

Substituting from Eq. (3.11 b) in the $p$-integrations on the right-hand side now gives,

$$
\begin{aligned}
\lambda \int \frac{p^{2} d p}{2 \pi^{2}} \frac{|g(p)|^{2}}{p^{2}-z} \bar{q}(p, P)= & \frac{1}{b(z)}-1 \\
& +\lambda \int \frac{k^{\prime 2} d k^{\prime}}{2 \pi^{2}} \frac{\left|g\left(k^{\prime}\right)\right|^{2}}{z-k^{\prime 2}} b\left(k^{\prime 2}\right) q\left(k^{\prime}, P\right)\left(\frac{1}{b(z)}-\frac{1}{b\left(k^{\prime 2}\right)}\right) .
\end{aligned}
$$

Finally, using also Eq. (3.11 a), gives the very important functional relation between $l(z)$ and $b(z)$, namely

$$
[b(z) l(z)]^{-1}=K(z) \equiv 1+\lambda \int_{0}^{\infty} \frac{k^{2} d k}{2 \pi^{2}} \frac{|g(k)|^{2}}{k^{2}-z}[\bar{q}(k, P)-q(k, P)] .
$$

This functional relation (3.12) may now be used to substitute back into our original coupled equations (3.11), and this not only effects the desired linearization but also as a bonus decouples the equations. The result is

$$
K(z) b(z)=1+\lambda \int_{0}^{\infty} \frac{k^{\prime 2} d k^{\prime}}{2 \pi^{2}} \frac{\left|g\left(k^{\prime}\right)\right|^{2}}{z-k^{\prime 2}} b\left(k^{\prime 2}\right) q\left(k^{\prime}, P\right)
$$




$$
K(z) l(z)=1+\lambda \int_{0}^{\infty} \frac{k^{2} d k}{2 \pi^{2}} \frac{|g(k)|^{2}}{k^{2}-z} l\left(k^{2}\right) \bar{q}(k, P) .
$$

The price that we have paid for this simplification is the production of equations (3.13) that are now singular in the physical regime. Thus the kernel of Eq. (3.13a) becomes singular for all physical values $z \rightarrow p^{\prime 2}$, since the energy denominator $\left(p^{\prime 2}-k^{\prime 2}\right)=\frac{1}{2}\left[\left|\frac{1}{2} \vec{P}+\vec{p}^{\prime}\right|^{2}+\left|\frac{1}{2} \vec{P}-\vec{p}^{\prime}\right|^{2}-\left|\frac{1}{2} \vec{P}+\vec{k}^{\prime}\right|^{2}-\left|\frac{1}{2} \vec{P}-\vec{k}^{\prime}\right|^{2}\right]$ can now vanish for all physical values of $p^{\prime 2}$, because $\vec{p}^{\prime}$ and $\vec{k}^{\prime}$ now have the same restrictions $\left|\frac{1}{2} \vec{P} \pm \vec{p}^{\prime}\right|<k_{F}$ and $\left|\frac{1}{2} \vec{P} \pm \vec{k}^{\prime}\right|<k_{F}$. Similar considerations apply to Eq. (3.13b). The solution of the remaining Eqs. (3.13) now rests upon the general theory developed by Muskhelishvili [9] and Omnès [10] for singular equations of the Hilbert type, and we first now show how Eqs. (3.13) may be brought into the standard Hilbert form.

From Eq. (3.12), by letting $z \rightarrow p^{2} \pm i \eta$, in the usual convention where $\eta$ henceforth represents a positive infinitesimal, it is easy to see that for $p$ real,

$$
\begin{aligned}
\operatorname{Im} K\left(p^{2} \pm i \eta\right) & =\lambda \int \frac{k^{2} d k}{2 \pi^{2}}|g(k)|^{2}( \pm \pi) \delta\left(k^{2}-p^{2}\right)[\bar{q}(k, P)-q(k, P)] \\
& = \pm \frac{\lambda}{4 \pi}|p||g(p)|^{2}[\bar{q}(p, P)-q(p, P)] .
\end{aligned}
$$

Inserting this result into Eqs. (3.13), and making use of the trivial observation from their definitions that the product $q(p, P) \bar{q}(p, P)=0$, it is easy to derive the results,

$$
\begin{aligned}
& K(z) b(z)=1 \pm \frac{1}{\pi} \int_{0}^{x_{0}^{2}} d k^{\prime 2} \frac{\operatorname{Im} K\left(k^{\prime 2} \pm i \eta\right) b\left(k^{\prime 2}\right)}{k^{\prime 2}-z} \\
& K(z) l(z)=1 \pm \frac{1}{\pi} \int_{x_{0}^{2}}^{\infty} d k^{2} \frac{\operatorname{Im} K\left(k^{2} \pm i \eta\right) l\left(k^{2}\right)}{k^{2}-z}
\end{aligned}
$$

where from Eqs. $(3.9 \mathrm{a}, \mathrm{b})$ we have defined $x_{0}$ by

$$
x_{0}^{2} \equiv\left(k_{F}^{2}-\frac{1}{4} P^{2}\right) \theta\left(2 k_{F}-P\right) .
$$

Making further use of Eq. (3.14) it is also now readily seen that in their respective physical regions, the functions $b$ and $l$ are explicitly real as required, i.e.

$$
\begin{array}{clrl}
b\left(p^{\prime 2} \pm i \eta\right) & =b\left(p^{\prime 2}\right), & & 0<p^{\prime 2}<x_{0}^{2} \\
l\left(p^{2} \pm i \eta\right) & =l\left(p^{2}\right), & & x_{0}^{2}<p^{2}<\infty
\end{array}
$$

Finally, defining new functions $\phi(z)$ and $\bar{\phi}(z)$ by

$$
\phi(z) \equiv K(z) b(z), \quad \bar{\phi}(z) \equiv K(z) l(z)
$$

we may rewrite Eqs. (3.15) in the standard Hilbert form

$$
\phi(z)=1+\frac{1}{i \pi} \int_{0}^{x_{0}^{2}} d k^{\prime 2} \frac{h\left(k^{\prime 2}\right) \phi\left(k^{\prime 2}+i \eta\right)}{k^{\prime 2}-z},
$$




$$
\bar{\phi}(z)=1+\frac{1}{i \pi} \int_{x_{0}^{2}}^{\infty} d k^{2} \frac{h\left(k^{2}\right) \bar{\phi}\left(k^{2}+i \eta\right)}{k^{2}-z},
$$

where for real values of $x^{2}$

$$
h\left(x^{2}\right) \equiv \frac{i \operatorname{Im} K\left(x^{2}+i \eta\right)}{K\left(x^{2}+i \eta\right)} .
$$

The equations (3.19) are now in the required standard form and may be solved by standard methods $[9,10]$ to give the general solutions as

$$
\begin{aligned}
& \phi(z)=1-\frac{e^{u(z)}}{2 \pi i} \int_{0}^{x_{0}^{2}} d k^{\prime 2} \frac{e^{-u\left(k^{\prime 2}+i \eta\right)}-e^{-u\left(k^{\prime 2}-i \eta\right)}}{k^{\prime 2}-z}+P(z) e^{u(z)}, \\
& \bar{\phi}(z)=1-\frac{e^{\bar{u}(z)}}{2 \pi i} \int_{x_{0}^{2}}^{\infty} d k^{2} \frac{e^{-\bar{u}\left(k^{2}+i \eta\right)}-e^{-\bar{u}\left(k^{2}-i \eta\right)}}{k^{2}-z}+\bar{P}(z) e^{\bar{u}(z)},
\end{aligned}
$$

where the functions $u(z)$ and $\bar{u}(z)$ are defined as

$$
\begin{aligned}
& u(z) \equiv \frac{1}{2 \pi i} \int_{0}^{x_{0}^{2}} d k^{\prime 2} \frac{\operatorname{Ln}\left[1 / G\left(k^{\prime 2}\right)\right]}{k^{\prime 2}-z}, \\
& \bar{u}(z) \equiv \frac{1}{2 \pi i} \int_{x_{0}^{2}}^{\infty} d k^{2} \frac{\operatorname{Ln}\left[1 / G\left(k^{2}\right)\right]}{k^{2}-z}
\end{aligned}
$$

with

$$
G\left(x^{2}\right) \equiv 1-2 h\left(x^{2}\right)
$$

and where the symbol Ln indicates any value of the multivalued logarithmic function continuous in the ranges $\left(0, x_{0}^{2}\right)$ and $\left(x_{0}^{2}, \infty\right)$ respectively. It should also be carefully noted that, as usual, the general solutions (3.20) to our singular equations are not unique since they still contain the unknown functions $P(z)$ and $\bar{P}(z)$ which are analytic functions of $z$ in the entire complex $z$-plane with the exception of singularities at their corresponding end-points of integration, namely $\left(0, x_{0}^{2}\right)$ and $\left(x_{0}^{2}, \infty\right)$ respectively.

At this point it is possible to simplify some of the expressions and to bring them into a more transparent form. Firstly, from Eqs. (3.20) and (3.23), we may write

$$
G\left(x^{2}\right)=1-2 i \frac{\operatorname{Im} K\left(x^{2}+i \eta\right)}{K\left(x^{2}+i \eta\right)}=\frac{K^{*}\left(x^{2}+i \eta\right)}{K\left(x^{2}+i \eta\right)}=e^{-2 i \delta\left(x^{2}+i \eta\right)}
$$

where the phase angle $\delta\left(x^{2}+i \eta\right)$ is real and defined by

$$
K\left(x^{2} \pm i \eta\right)=\left|K\left(x^{2} \pm i \eta\right)\right| e^{ \pm i \delta\left(x^{2}+i \eta\right)}
$$

or, equivalently, by

$$
\delta\left(x^{2}+i \eta\right)=\operatorname{Tan}^{-1}\left(\frac{\operatorname{Im} K\left(x^{2}+i \eta\right)}{\operatorname{Re} K\left(x^{2}+i \eta\right)}\right)
$$

and where, consistent with the Ln function in Eqs. (3.21), the symbol $\operatorname{Tan}^{-1}$ now 
indicates any value of the multivalued inverse tangent function which is continuous over the respective integration intervals. We thus have the important relations,

$$
\begin{aligned}
& u(z)=\frac{1}{\pi} \int_{0}^{x_{0}^{2}} d k^{\prime 2} \frac{\delta\left(k^{\prime 2}+i \eta\right)}{k^{\prime 2}-z}, \\
& \bar{u}(z)=\frac{1}{\pi} \int_{x_{0}^{2}}^{\infty} d k^{2} \frac{\delta\left(k^{2}+i \eta\right)}{k^{2}-z} .
\end{aligned}
$$

For convenience and in order to be definite henceforth, we now consistently choose to work on that branch of the phase function $\delta$ which is zero at infinity. In this case both $u(z)$ and $\bar{u}(z)$ tend to zero at infinity. Further simplification of the integrals in Eqs. (3.21) is now possible by considering the contour integrals of the functions $\exp \left[-u\left(z^{\prime}\right)\right] /\left(z^{\prime}-z\right)$ and $\exp \left[-\bar{u}\left(z^{\prime}\right)\right] /\left(z^{\prime}-z\right)$ respectively with respect to the variable $z^{\prime}$ around the contour comprising the circle at infinity indented to exclude the positive real axis. Comparison of direct evaluation with the result obtained using Cauchy's residue theorem easily leads to the final general solutions of the singular equations (3.15),

$$
b(z)=\frac{e^{u(z)}}{K(z)}[1+P(z)], \quad l(z)=\frac{e^{\bar{u}(z)}}{K(z)}[1+\bar{P}(z)] .
$$

At this point, it only remains therefore to eliminate (as far as possible) the unknown functions $P(z)$ and $\bar{P}(z)$. Of great assistance here is the fact that our solutions are still required to satisfy the original (nonlinear) Eqs. (3.7), and more particularly also the functional relation (3.12) derived from them. Inserting from Eq. (3.27) into Eq. (3.12) yields the relation

$$
[1+P(z)][1+\bar{P}(z)]=K(z) \exp \{-[u(z)+\bar{u}(z)]\},
$$

which, in passing, demonstrates that the functions $[1+P(z)]$ and/or $[1+\bar{P}(z)]$ must have the same zeros as the function $K(z)$. Equation (3.28) may now be rewritten in more useful form by using Eqs. (3.26) and (3.25a),

$$
[1+P(z)][1+\bar{P}(z)]=\exp \left[\operatorname{Ln} K(z)-\frac{1}{2 \pi i} \int_{0}^{\infty} d x^{2} \frac{\operatorname{Ln} K\left(x^{2}+i \eta\right)-\operatorname{Ln} K\left(x^{2}-i \eta\right)}{x^{2}-z}\right] \text {. }
$$

The integral term on the right-hand side may now be simplified by considering the contour integral $I(z)$,

$$
I(z) \equiv \frac{1}{2 \pi i} \oint_{C} d z^{\prime} \frac{\operatorname{Ln} K\left(z^{\prime}\right)}{z^{\prime}-z}
$$

where again we work with the same branch of the multivalued Ln function that is continuous over the contour $C$. The contour $C$ is chosen to comprise (i) the circle at infinity (which gives no contribution with our chosen branch), with suitable indentations to exclude both (ii) the whole of the positive real axis $z^{\prime} \rightarrow x^{2}$ since this is the (only) branch cut of $K(z)$ and (iii) all additional branch cuts of $\operatorname{Ln} K(z)$ which can only arise from any zeros in the complex $z$-plane of the function $K(z)$. In this case, the function $\operatorname{Ln} K\left(z^{\prime}\right)$ is analytic everywhere inside the contour $C$ and $I(z)$ is immediately 
evaluated as $\operatorname{Ln} K(z)$ by Cauchy's theorem. Comparison with direct evaluation then enables us to write Eq. (3.29) as

$$
[1+P(z)][1+\bar{P}(z)]=\exp \left[-\sum_{i} \frac{1}{2 \pi i} \oint_{\Gamma_{i}} d z^{\prime} \frac{\operatorname{Ln} K\left(z^{\prime}\right)}{z^{\prime}-z}\right],
$$

where the contours $\Gamma_{i}$ surround (in the usual anticlockwise sense) each of the branches $B_{i}$ arising purely from any zeros in the $K$-function. In this case, choosing the contours $\Gamma_{i}$ to be infinitesimally close either side of the branches $B_{i}$, the discontinuity across the branch $B_{i}$ will be $2 \pi i n_{i}$ for some (positive or negative) integer $n_{i}$. If we choose $a_{i}$ and $b_{i}$ to be the end-points of the branches $B_{i}$, then Eq. (3.31) reduces to

$$
[1+P(z)][1+\bar{P}(z)]=\prod_{i}\left(\frac{a_{i}-z}{b_{i}-z}\right)^{n_{i}} .
$$

The exact arrangement of the branch cuts $B_{i}$ depends in each case on the particular branch of $\delta(z)$ or equivalently of $\operatorname{Ln} K(z)$ which we have used. In each case, however, at least one of the end-points $a_{i}$ or $b_{i}$ must (by definition) be one of the zeros of $K(z)$. The other end-point may be either another zero of $K(z)$ or one of the remaining branch-points $\left(0, x_{0}^{2}\right)$ of $K(z)$.

It is clear at this point that any possible zeros in the function $K(z)$ now play a very crucial role in our discussion, and it is these as we shall see later that correspond precisely to the energies of the "bound pairs" in the medium in this approximation. The zeros in the $K$-function of Eq. (3.12) will depend specifically upon the particular form of the form factor $g(k)$ and on the strength $\lambda$ of the interaction, and particularly on whether the potential is "repulsive" $(\lambda>0)$ or "attractive" $(\lambda<0)$. Even for a specific potential the $K$-function still depends both on the total momentum $P$ of the interacting pair and on the density $\rho$, or equivalently the Fermi momentum $k_{F}$, of the system. In this present work we are less interested in the details of the calculation for a specific system than to enumerate the main possibilities that can arise in practice, without specifying a particular form for the potential. Since we are specifically concerned here with the particular problem of pairing we restrict ourselves mainly to a discussion of the case $\lambda<0$. We defer to the Appendix a discussion of the various possibilities that can arise for the zeros in the $K$-function, and continue in this section to use the results obtained in the Appendix to demonstrate how to calculate explicitly the g.s. wavefunction and energy in some of the various cases that can arise.

Before proceeding with some particular cases however, it is of some interest to use our solution to re-express the g.s. energy given by Eq. (3.10). Using the starting integral equation ( 3.13 a) for $b\left(p^{2}\right)$ it is easy to see that

$$
\lambda \int \frac{p^{\prime 2} d p^{\prime}}{2 \pi^{2}}\left|g\left(p^{\prime}\right)\right|^{2} b\left(p^{\prime 2}\right) q\left(p^{\prime}, P\right)=\lim _{z \rightarrow \infty}\{z[K(z) b(z)-1]\},
$$

which allows us to rewrite the interaction term in Eq. (3.10) accordingly. This may be further simplified by using the general solution from Eq. (3.27) to write

$$
K(z) b(z)-1=e^{u(z)}[1+P(z)]-1 .
$$

Taking the limit $z \rightarrow \infty$ now yields the result 
$\frac{E}{N}=\frac{3}{5} \frac{\hbar^{2} k_{F}^{2}}{2 m}+\frac{3}{2} \frac{\hbar^{2}}{m k_{F}^{3}} \int_{0}^{2 k_{F}} P^{2} d P \lim _{z \rightarrow \infty}\left\{\left[z-\frac{1}{\pi} \int_{0}^{x_{0}^{2}} d k^{\prime 2} \delta\left(k^{\prime 2}+i \eta\right)\right][1+P(z)]-z\right\}$,

where we have used the definition (3.25a) and expanded the exponential.

Thus, in order to calculate the exact CLAD g.s. wavefunctions and energies, all that remains is to evaluate the functions $P(z)$ and $\bar{P}(z)$. As we have so far attempted to treat the problem as generally as possible by not introducing an explicit expression for the form factor $g(k)$ we cannot perform any of the remaining integrals, in Eq. (3.33) for example. However, by substituting the expressions obtained below for $[1+P(z)]$ using the results obtained in the Appendix for various cases, we may also put the energy expression (3.33) into forms which allow us to make some definite deductions about the structure of the g.s. obtained by our g.s. CCF for these cases.

In order to be specific we now consider the particular case which is physically most interesting, namely the case of an attractive potential $(\lambda<0)$ which is strong enough to bind a free (i.e., in vacuo) pair of particles. As detailed in the Appendix (to which the reader should now refer), there are three main possibilities in this case. At least at small enough values of the total momentum $P$, there is first a range of densities $0<k_{F}<k_{F m}$ for which the real negative zero at $z=-\kappa_{1}^{2}$ in $K(z)$ corresponds to the free bound state becoming progressively less bound when immersed in the many-body medium. Secondly, there is a density range specified by $k_{F m}<k_{F}<k_{F M}$ in which a second real zero appears at $z=-\kappa_{2}^{2}$, with $\kappa_{2}<\kappa_{1}$. In this range there are thus two "bound states", the former remaining predominantly particle-particle like and the new one (which has no counterpart either in the free case or in the BG approximation) being predominantly hole-hole like. Lastly, there is a range of densities corresponding to $k_{F}>k_{F M}$ for which the two previous zeros merge at $k_{F} \rightarrow k_{F M}$ and then move into the left-half complex plane as conjugate pairs, and which we therefore expect to manifest themselves physically as resonance/antiresonance pairs. This behaviour is illustrated in Fig. 3 of the Appendix. (For increasing values of the total momentum $P$, these "bound states" become progressively less bound until for given density there is a critical value of $P$ above which they disappear.) The behaviour of the phase angle $\delta(z)$ is considered in the Appendix in some detail for these three cases (referred to there as Cases I, II and III, respectively), and is shown in particular in Figs. 5-7 respectively for $z \rightarrow x+i \eta$. We therefore now discuss these three particular cases in turn, referring to the Appendix for the necessary details, and noting that the remaining cases of weakly attractive potentials (not able to support a bound pair in vacuo) and repulsive potentials may be handled in an essentially similar manner.

Case $I$ (strong attraction, i.e. sufficient to bind a pair in vacuo; with $0<k_{F}<k_{F m}$ ). In this case the only zero in $K(z)$ is at the real negative value $z=-\kappa_{1}^{2}$, and as considered above in the discussion leading to Eqs. (3.31)-(3.32), our derivation of the solution may be completed by considering the contour $C$ in the integral of Eq. (3.30) to comprise in this case the circle at infinity indented only to circumnavigate (infinitesimally above and below) the entire real axis. Working on the branch of the phase angle $\delta(z)$ indicated in Fig. 5 for this case, it is now simple to show that Eq. (3.32) reduces in this Case I to 


$$
[1+P(z)][1+\bar{P}(z)]=\frac{z+\kappa_{1}^{2}}{z} .
$$

All that thus remains is for us to decide how the factors on the right-hand side of Eq. (3.34 a) actually divide between the two factors on the left. First we recall that the functions $b(z)$ and $l(z)$ given by Eq. (3.27) must (in order to satisfy the functional relation (3.12)) be real, non-singular, and non-zero over the ranges for which they are "physical", namely $b(z) \rightarrow b\left(p^{\prime 2}\right)$ with $0 \leqslant p^{\prime 2} \leqslant x_{0}^{2}$, and $l(z) \rightarrow l\left(p^{2}\right)$ with $p^{2} \geqslant x_{0}^{2}$, where $x_{0}$ is defined in Eq. (3.16). Now, from the definitions (3.26) of the functions $u(z)$ and $\bar{u}(z)$ and from our sketch of the phase angle $\delta\left(x^{2}+i \eta\right)$ given in Fig. 5 of the Appendix for this case, we may readily verify the limiting forms below,

$$
\begin{array}{ll}
e^{u(z)} \underset{z \rightarrow 0}{\longrightarrow} \text { (const.) } z, & e^{u(z)} \underset{z \rightarrow x_{0}^{2}}{\longrightarrow} \frac{\text { const. }}{z-x_{0}^{2}}, \\
e^{\bar{u}(z)} \underset{z \rightarrow x_{0}^{2}}{\longrightarrow} \text { const. }\left(z-x_{0}^{2}\right), & e^{\bar{u}(z)} \underset{z \rightarrow \infty}{\longrightarrow} 1 .
\end{array}
$$

This information is now precisely sufficient to allow us to determine the factors $[1+P(z)]$ and $[1+\bar{P}(z)]$ up to an arbitrary multiplicative constant factor $\alpha$, $\alpha^{-1}$ respectively (where $\alpha$ can itself be fixed by observing that both $b(z)$ and $l(z)$ approach unity asymptotically as $z \rightarrow \infty$ from their original defining equations (3.11)), when we also recall that $P(z)$ and $\bar{P}(z)$ may have singularities only at the respective end-points $\left(0, x_{0}^{2}\right)$ and $\left(x_{0}^{2}, \infty\right)$ of their "physical" ranges. Thus we have from Eqs. (3.34 a) and (3.35 a) the final results,

$$
1+P(z)=\frac{z-x_{0}^{2}}{z}, \quad 1+\bar{P}(z)=\frac{z+\kappa_{1}^{2}}{z-x_{0}^{2}},
$$

and hence the full (and unique) solutions for this Case I,

$$
b(z)=\left(1-\frac{x_{0}^{2}}{z}\right) \frac{e^{u(z)}}{K(z)}, \quad l(z)=\left(\frac{z+\kappa_{1}^{2}}{z-x_{0}^{2}}\right) \frac{e^{\bar{u}(z)}}{K(z)} .
$$

Finally, we can also use the solution (3.36a) to rewrite the expression (3.33) for the energy per particle of the many-body system in this case in the form

$\frac{E}{N}=\frac{3}{5} \frac{\hbar^{2} k_{F}^{2}}{2 m}+\frac{3}{2} \frac{\hbar^{2}}{m k_{F}^{3}} \int_{0}^{2 k_{F}} P^{2} d P\left\{-\frac{1}{\pi} \int_{0}^{x_{0}^{2}} d k^{\prime 2}\left[\pi+\delta\left(k^{\prime 2}+i \eta\right)\right]\right\}$.

We note only for the moment that from the plot of $\delta\left(x^{2}+i \eta\right)$ given in Fig. 5 in this Case I, the contribution to the potential energy from pairs of total momentum $P$ (and at densities corresponding to the range $0<k_{F}<k_{F m}$ under consideration) is positive-definite even though the potential is attractive. We shall leave a more detailed discussion of this important point until Sect.4, after we consider the remaining Cases II and III.

Case II (strong attraction, i.e. sufficient to bind a pair in vacuo; with $\left.k_{F m}<k_{F}<k_{F M}\right)$. In this case the only zeros in $K(z)$ are at the real negative values $z=-\kappa_{1}^{2}$ and $z=-\kappa_{2}^{2}$, and otherwise the discussion proceeds exactly as in Case I. Working now on the branch of the phase angle $\delta(z)$ indicated in Fig. 6 for this case, a comparable analysis shows that Eq. (3.31) reduces to 


$$
[1+P(z)][1+\bar{P}(z)]=\frac{\left(z+\kappa_{1}^{2}\right)\left(z+\kappa_{2}^{2}\right)}{z^{2}} .
$$

In this case, use of Eq. (3.26) and the phase angle shown in Fig. 6, leads to the limiting forms

$$
\begin{array}{ll}
e^{u(z)} \underset{z \rightarrow 0}{\longrightarrow} \text { (const.) } z^{2}, & e^{u(z)} \underset{z \rightarrow x_{0}^{2}}{\longrightarrow} \frac{\text { const. }}{z-x_{0}^{2}}, \\
e^{\bar{u}(z)} \underset{z \rightarrow x_{0}^{2}}{\longrightarrow} \text { const. }\left(z-x_{0}^{2}\right), & e^{\bar{u}(z)} \underset{z \rightarrow \infty}{\longrightarrow} 1 .
\end{array}
$$

Similar considerations to Case I now again lead from Eqs. (3.34 b) and (3.35 b) to the final results,

$$
1+P(z)=\frac{\left(z-x_{0}^{2}\right)\left(z+\kappa_{2}^{2}\right)}{z^{2}}, \quad 1+\bar{P}(z)=\frac{z+\kappa_{1}^{2}}{z-x_{0}^{2}},
$$

and hence to the full solutions for this Case II,

$$
b(z)=\left(1-\frac{x_{0}^{2}}{z}\right)\left(1+\frac{\kappa_{2}^{2}}{z}\right) \frac{e^{u(z)}}{K(z)}, \quad l(z)=\left(\frac{z+\kappa_{1}^{2}}{z-x_{0}^{2}}\right) \frac{e^{\bar{u}(z)}}{K(z)} .
$$

We note here that the only non-uniqueness that enters the expressions ( $3.36 \mathrm{~b})$ and $(3.37 \mathrm{~b})$ is the possibility of interchanging the roles of $\kappa_{1}$ and $\kappa_{2}$. The decision to write the final expressions in this particular definite form rests (only) on an appeal to continuity with Case I at $k_{F} \rightarrow k_{F m}$. Finally we may also use the solution (3.36 b) to rewrite the expression (3.33) for the energy per particle in this case, as

$$
\frac{E}{N}=\frac{3}{5} \frac{\hbar^{2} k_{F}^{2}}{2 m}+\frac{3}{2} \frac{\hbar^{2}}{m k_{F}^{3}} \int_{0}^{2 k_{F}} P^{2} d P\left\{-\frac{1}{\pi} \int_{0}^{x_{0}^{2}} d k^{\prime 2}\left[\pi+\delta\left(k^{\prime 2}+i \eta\right)\right]+\kappa_{2}^{2}\right\} .
$$

Once again, inspection of Fig. 6 for this Case II shows that the potential energy contribution from Case II pairs is positive-definite.

Case III (strong attraction, i.e. sufficient to bind a pair in vacuo; with $k_{F}>k_{F M}$ and "resonance pairs"). In this case the two real zeros in $K(z)$ have first coalesced as $k_{F} \rightarrow k_{F M}$ and have then moved into the complex plane as the conjugate pair at $z=-\kappa_{R}^{2} \pm i \Gamma_{R}$ for $k_{F}$ increasing through $k_{F M}$. Drawing the consequent branch cut in $\operatorname{Ln} K(z)$ as the straight line joining these zeros (as assumed in Fig. 7 for this case), our solution is now completed by considering the contour $C$ in the integral of Eq. (3.30) to comprise the circle at infinity indented only to circumnavigate (infinitesimally closely) both the entire real axis and the branch cut joining these zeros. Working now on the branch of the phase angle $\delta(z)$ indicated in Fig. 7 for this case, Eq. (3.31) reduces in this Case III to

$$
[1+P(z)][1+\bar{P}(z)]=\frac{\left(z+\kappa_{R}^{2}+i \Gamma_{R}\right)\left(z+\kappa_{R}^{2}-i \Gamma_{R}\right)}{z^{2}} .
$$

Again, use of Eq. (3.26) with the phase angle of Fig. 7 leads to the same limiting forms (3.35 b) for this Case III as for Case II, and hence to the final results, 


$$
1+P(z)=\frac{\left(z-x_{0}^{2}\right)\left(z+\kappa_{R}^{2}-i \Gamma_{R}\right)}{z^{2}}, \quad 1+\bar{P}(z)=\frac{z+\kappa_{R}^{2}+i \Gamma_{R}}{z-x_{0}^{2}}
$$

for the functions $P(z)$ and $\bar{P}(z)$, and finally also to the full solutions for this Case III,

$$
b(z)=\left(1-\frac{x_{0}^{2}}{z}\right)\left(1+\frac{\kappa_{R}^{2}-i \Gamma_{R}}{z}\right) \frac{e^{u(z)}}{K(z)}, \quad l(z)=\left(\frac{z+\kappa_{R}^{2}+i \Gamma_{R}}{z-x_{0}^{2}}\right) \frac{e^{\bar{u}(z)}}{K(z)} .
$$

The only possible non-uniqueness remaining in our solution for this Case III is the possibility of changing everywhere in Eqs. (3.36c) and $(3.37 \mathrm{c})$ the sign of the imaginary part $\Gamma_{R}$ of the "resonance" energy, which displays the usual symmetry between conjugate resonance/anti-resonance pairs. Physical boundary conditions must ultimately decide the issue by the usual choice of the exponentially decaying state. Finally in this case, the energy per particle expression (3.33) may now be rewritten, using the solution $(3.36 \mathrm{c})$ as

$\frac{E}{N}=\frac{3}{5} \frac{\hbar^{2} k_{F}^{2}}{2 m}+\frac{3}{2} \frac{\hbar^{2}}{m k_{F}^{3}} \int_{0}^{2 k_{F}} P^{2} d P\left\{-\frac{1}{\pi} \int_{0}^{x_{0}^{2}} d k^{\prime 2}\left[\pi+\delta\left(k^{\prime 2}+i \eta\right)\right]+\kappa_{R}^{2}-i \Gamma_{R}\right\}$.

We note only that the total energy is now complex, as expected, and that inspection of Fig. 7 for this Case III shows as in the previous two cases that the real part of the potential energy contribution from Case III pairs is positive-definite.

We defer a discussion of our now complete analytical solutions to Sect. 4 , but for purposes of comparison we end this section with a brief discussion of the (almost trivial) analogous solution for the case of the BG approximation.

\subsection{The ppLAD (BG) Approximation}

The Bethe-Goldstone (BG) equation with bare kinetic energies in the intermediate two-body propagators, may be obtained by further ignoring in the complete ladder (CLAD) summation the hole-hole and mixed particle-particle and hole-hole scattering ladder terms represented diagrammatically by the terms $(d)$ and $(e)$ in Fig. 1 of $I$. This corresponds equivalently to ignoring the last two terms on the lefthand side of Eq. (2.16) of I. The resulting ppLAD approximation then gives the analogue of the BG equation for the two-body correlation operator $S_{2}$ of the CCF as

$$
\begin{aligned}
& \frac{\hbar^{2}}{m}\left(p^{2}-p^{\prime 2}\right) S_{2 ; \vec{p} \vec{p}^{\prime}}^{s_{1} s_{2}}(\vec{P})+\left\langle\vec{p}|V| \vec{p}^{\prime}\right\rangle-\left\langle\vec{p}|V|-\vec{p}^{\prime}\right\rangle \delta_{s_{1} s_{2}} \\
& +\sum_{\vec{k}}\langle\vec{p}|V| \vec{k}\rangle S_{2 ; \vec{k} \vec{p}^{\prime}}^{s_{1} s_{2}}(\vec{P}) \bar{Q}(\vec{k}, \vec{P})=0 .
\end{aligned}
$$

Once again the solution to the BG equation (3.39) may be written in explicitly antisymmetric form as

$$
S_{2 ; \vec{p} \vec{p}^{\prime}}^{S_{1} S_{2}}(\vec{P})=T_{\vec{p} \vec{p}^{\prime}}^{\mathrm{BG}}(\vec{P})-\delta_{s_{1} S_{2}} T_{\vec{p},-\vec{p}^{\prime}}^{\mathrm{BG}}(\vec{P}) .
$$

Proceeding to the thermodynamic limit and working in the same plane-wave basis as in the CLAD approximation, we have trivially that $T^{\mathrm{BG}}$ satisfies the equation 


$$
\frac{\hbar^{2}}{m}\left(p^{2}-p^{\prime 2}\right) T_{\vec{p} \vec{p}^{\prime}}^{\mathrm{BG}}(\vec{P})=-\left\langle\vec{p}|V| \vec{p}^{\prime}\right\rangle-\Omega \int \frac{d \vec{k}}{(2 \pi)^{3}}\langle\vec{p}|V| \vec{k}\rangle T_{\vec{k} \vec{p}^{\prime}}^{\mathrm{BG}}(\vec{P}) \bar{Q}(\vec{k}, \vec{P})
$$

With the same one-term, $S$-wave, separable potential (3.3) as before, Eq. (3.41) is now trivially solved as

$$
T_{\vec{p} \vec{p}^{\prime}}^{\mathrm{BG}}(\vec{P})=-\frac{\lambda}{\Omega} \frac{g(p) g^{*}\left(p^{\prime}\right)}{p^{2}-p^{\prime 2}} \frac{1}{K_{\mathrm{BG}}\left(p^{\prime 2}\right)},
$$

where

$$
K_{\mathrm{BG}}(z) \equiv 1+\lambda \int_{x_{0}^{2}}^{\infty} \frac{k^{2} d k}{2 \pi^{2}} \frac{|g(k)|^{2}}{k^{2}-z} \bar{q}(k, P) .
$$

Comparisons of the CLAD solution (3.5) with the ppLAD solution (3.42) and also of the corresponding $K$-functions defined in Eqs. (3.12) and (3.43), then show that formally the ppLAD solution may be obtained from the CLAD solution by the replacements

$$
\begin{aligned}
l(z) & \rightarrow l_{\mathrm{BG}}(z) \equiv 1, \\
b(z) & \rightarrow b_{\mathrm{BG}}(z) \equiv 1 / K_{\mathrm{BG}}(z), \\
K(z) & \rightarrow K_{\mathrm{BG}}(z) .
\end{aligned}
$$

With the solution given by Eqs. (3.40), (3.42) and (3.43), the BG total energy may also be evaluated from Eq. (2.13) as

$$
\begin{aligned}
E_{\mathrm{BG}}= & \frac{3}{5} \frac{\hbar^{2} k_{F}^{2}}{2 m} N+\Omega \lambda \frac{\hbar^{2}}{m} \int \frac{p^{\prime 2} d p^{\prime}}{2 \pi^{2}} \int \frac{P^{2} d P}{2 \pi^{2}} q\left(p^{\prime}, P\right)\left|g\left(p^{\prime}\right)\right|^{2} \\
& \times\left[1-\frac{\lambda}{K_{\mathrm{BG}}\left(p^{\prime 2}\right)} \int \frac{p^{2} d p}{2 \pi^{2}} \frac{|g(p)|^{2}}{p^{2}-p^{\prime 2}} \bar{q}(p, P)\right] .
\end{aligned}
$$

Using Eq. (3.43) enables us to write the g.s. energy per particle in the BG approximation in the final form,

$\frac{E_{\mathrm{BG}}}{N}=\frac{3}{5} \frac{\hbar^{2} k_{F}^{2}}{2 m}+\frac{3}{2} \lambda \frac{\hbar^{2}}{m k_{F}^{3}} \int_{0}^{2 k_{F}} P^{2} d P \int_{0}^{x_{0}^{2}} \frac{p^{\prime 2} d p^{\prime}}{2 \pi^{2}} \frac{\left|g\left(p^{\prime}\right)\right|^{2}}{K_{\mathrm{BG}}\left(p^{\prime 2}\right)} q\left(p^{\prime}, P\right)$.

Comparison of the CLAD and ppLAD expressions (3.10) and (3.45), respectively, again shows that the latter may be formally derived from the former by the same replacements (3.44) above. The BG energy expression (3.45) may similarly also be written for the various BG cases in terms of the corresponding phase angle $\delta_{\mathrm{BG}}$ of $K_{\mathrm{BG}}$, in analogy with Eqs. (3.38) for the various CLAD cases.

\section{Discussion and Conclusions}

From the results derived in Sect. 3 for the CLAD functions $l(z)$ and $b(z)$ and the corresponding g.s. energies, it is possible to draw certain conclusions concerning the g.s. structure. The important points may be summarised as follows. 
For densities corresponding to $0<k_{F}<k_{F m}$ where the potential is assumed capable of supporting a bound state in vacuo, the "bound state" zero manifests itself [and see Eq. (3.37a)] in that part of the wavefunction called $l(z)$ in our notation which in the physical scattering regime corresponds to two particles outside the unperturbed Fermi sea. As a consequence we have seen that this bound state does not manifestly contribute to the g.s. energy expression (3.38 a). This alone is clear evidence that the so-called g.s. obtained within our so-called g.s. CCF within which we have been working, is an uncondensed fluid-like phase, with the bound pairs of binding energy $\hbar^{2} \kappa_{1}^{2} / m$ remaining unrealised possibilities. We expect the formation of these bound pairs to give rise to what must be treated therefore as excited states within this formalism. Clearly the term "excited state" here is something of a misnomer since the formation of such real bound pairs will clearly lower the energy of the system, and hence perhaps the term "de-excited state" would be more apposite.

For densities corresponding to $k_{F m}<k_{F}<k_{F M}$ the second zero in $K(z)$ at $z=-\kappa_{2}^{2}$ now appears in that part of the two-body cluster wavefunction, $b(z)$, which in the physical scattering regime describes two holes interacting inside the Fermi sea, while the other zero corresponding to the more bound state at $z=-\kappa_{1}^{2}$ appears in $l(z)$ as before [and see Eq. $(3.37 \mathrm{~b})$ ]. As we have seen in this case from Eq. (3.38 b) the g.s. energy now manifestly acquires a positive contribution from what are now the predominantly hole-hole-like pairs of bound quasiparticles within the many-body medium of binding energy $\hbar^{2} \kappa_{2}^{2} / m$, but conversely again the g.s. energy has no contribution from the predominantly particle-particle-like pairs of binding energy $\hbar^{2} \kappa_{1}^{2} / m$. It would thus seem that the so-called g.s. given by the $\mathrm{CCF}$ in this case does contain bound hole-hole-like pairs (which however increase the energy) but no bound particle-particle-like pairs. Again we conclude that the formation of the latter, whose existence in this density region is proven, must manifest itself in the (de-)excited state spectrum of the system within the CCF.

Finally for densities corresponding to $k_{F}>k_{F M}$ the two previously real bound states have moved into the complex energy plane as conjugate resonanceantiresonance pairs. In this case the resonant state, say, manifests itself in the function $b(z)$ whose physical scattering regime corresponds to real hole-hole interactions inside the Fermi sea, and conversely the antiresonant state manifests itself in the function $l(z)$ whose physical scattering regime corresponds to real particle-particle interactions outside the Fermi sea [and see Eq. (3.37 c)]. In this case the g.s. energy duly becomes complex as expected, as an indication of the finite lifetime of the resonant states. The real part of the energy, however, is again manifestly increased [and see Eq. $(3.38 \mathrm{c})$ ] when the real part of the resonant pair energy is negative. Thus again the g.s. obtained within the CCF does not contain the extra binding due to the possible formation of the (admittedly unstable) resonant bound pairs; and we again therefore expect a comparable (de-)excited state to exist accordingly in the excited-state CCF.

In order to verify these assertions concerning the (de-)excited states of the system (and indeed also really to prove that the zeros of the $K$-function actually do correspond to composite pair states, as we have consistently asserted from the very suggestive manner in which they arise in this g.s. CCF), it is clearly of considerable interest to formulate a treatment at this same CLAD level of approximation, within 
the excited-state CCF due to Emrich $[11,12]$. Suffice it to say here that this is done in a subsequent paper [13] by the present authors.

In conclusion we note that our main aim in I and in this work has been to formulate the complete ladder (CLAD) summation within the CCF, i.e. the CLAD sub-approximation to the SUB2 approximation of the g.s. CCF. As we have pointed out, the physical approximations involved here are completely equivalent to those made in the Galitskii-Feynman approximation in the Green's function formalism of time-dependent perturbation theory. In order to make analytic progress we have discussed a simple many-body system, in which the constituent particles interact via an $S$-wave, pairwise, one-term separable potential, and which can be solved exactly at this level of approximation. The basic CLAD equation for the two-body correlations, or equivalently for the two-particle/two-hole wavefunction, was shown to be exactly equivalent to a pair of highly non-trivial, coupled, nonlinear integral equations. We succeeded in simultaneously exactly decoupling and linearising these equations, being guided by the remarkable similarity which exists between the various "ring" (or "bubble") approximations for the Fermi plasma with local (e.g., Coulomb) two-body interactions and the various generalised "ladder" approximations for (non-local) separable two-body interactions. In this way we reduced our coupled nonlinear equations to a pair of linear, but singular, integral equations of the Hilbert-Muskhelishvili-Omnès type. We showed further that a complete solution of these equations rests intimately upon a detailed knowledge of the analytic structure of the function $\operatorname{Ln} K(z)$ which we discuss in some detail in the Appendix. We derived exact solutions for the many-body wavefunction and its corresponding energy within the CLAD approximation, for several cases of interest when the potential is attractive. We showed further how these solutions give strong indications of the structure both of the uncondensed so-called ground state obtained within our so-called g.s. CCF, and also of the so-called (de-)excited states. These results also suggested that an examination of the so-called excited state $\mathrm{CCF}$ would be necessary and of considerable interest in its own right, in order to confirm our suspicions regarding the role of the zeros in the $K$-function as representing the energies of the various composite-pair states, and also how these actually manifest. themselves in our CCF many-body states.

What we have seen in this method of discussion based on the assumption of a uniform fluid-like (i.e., a fluid of the constituent particles, not of their composites) ground state, is that the formation of such bound pair states gives rise to the (de-)excited states of the system. We have seen that the g.s. CCF already gives a considerable amount of information on the structure of these (de-)excited states. Indeed one could see very clearly that they are contained in our g.s. calculation as "virtual (de-)excitations". It is clear, however, that the formation of bound pairs in a many-body system must physically lower its energy, and hence as we have seen our (de-)excited states will have lower energy than our fluid-like ground state obtained here. This is indeed borne out in ref. [13]. We might legitimately ask, however, how this situation has arisen. Its origin clearly lies in our choice of model state $|\Phi\rangle$ to be itself an uncondensed fluid-like state (namely, the unperturbed g.s. or filled Fermi sea of plane-wave states). In this case the socalled g.s. CCF employed here generates the state $|\Psi\rangle$ (of lowest energy) that by construction has non-zero overlap with $|\Phi\rangle$, since from Eqs. (2.6) and (2.7), $\langle\Phi \mid \Psi\rangle=1$. By contrast, the so-called excited-state formalism of Emrich 
$[11,12]$ is constructed to describe those ("excited") states $\left|\Psi_{e}\right\rangle$ which are orthogonal to $|\Phi\rangle$. The states involving composite pairs that we have observed have essentially different symmetries to, and hence zero overlap with $|\Phi\rangle$, and so naturally arise within this formalism as (de-)excited states in our approach. The interested reader may also be referred to a fuller discussion of these points by Kümmel [14]. The problem is in essence therefore one of nomenclature alone (and indeed, one that may not arise with a different choice of model state $|\Phi\rangle$ ).

There are several more points worth noting about the calculations presented here. We have kept the discussion as general as possible by not referring to any specific form for the separable potential. In so doing (and by comparison with previous numerical calculations [15-18] using local potentials) we believe that we have enumerated all of the various general possibilities that may arise for the singularities of the function $\operatorname{Ln} K(z)$. In the process we have discovered in particular the necessity of resonance states which to our knowledge have not previously been mentioned in this context. For application to a specific form of separable interaction, such as the commonly used Yamaguch type [19], the analysis presented here can be immediately put into effect. It is still possible that other special structures may exist, with consequent physical implications, for such special forms of the separable form factor as those suggested by Tabakin [20] (and as further referred to in the Appendix) to enable both repulsion and attraction to be represented in the one-term separable form. However, even in such cases our general analysis is still applicable with obvious modifications.

We should perhaps also comment on the obvious fact that by comparison with, for example, the Green's function techniques that for the present problem are almost trivial for calculations of the g.s. energy, our g.s. CCF is very much more cumbersome and involves much more complicated mathematics (necessarily nonlinear in the case of the CLAD approximation even). If one was only interested in the g.s. energy it is clear that the CCF would hardly be the method of first choice. However, there are two salient points worth making in this regard. Firstly we stress that our CCF solution has provided us with much more information in the form of real two-body wavefunctions in the physical scattering regime, which the GF formalism using Green's function techniques do not give. These wavefunctions are of course not vital for the g.s. energy but are certainly needed to calculate expectation values or other matrix elements of any other operator. They are even more useful, as we show in a later paper [13], for a calculation of the wavefunctions describing the actual composite pairs (as (de-)excited states) within the many-body medium. Secondly we note that while the ladder approximation reduces in the GF formalism to a linear equation (and indeed for separable potentials to just an algebraic equation), the moment one tries to improve upon the ladder approximation (for example by including the equivalent effects of other neglected terms in the full SUB2 approximation) this ceases to be the case. Thus for further calculations the GF method instantly loses what we now argue to be a rather artificial advantage it has in this very special limit, and the full power of the CCF becomes apparent, as has been discussed more fully elsewhere $[6,7]$.

We note also that while the one-term separable interaction employed here is clearly rather artificial, there are three main points that may be made in its defence. Firstly we note that the method presented here readily lends itself to the generalisations to include other partial waves and/or multi-term separable poten- 
tials in a given partial wave. With these generalisations one can model even a local potential rather accurately. Secondly, and more importantly, we note that considering only the various types of composite pairing observed here, the one-term separable potential model displays qualitatively exactly the same behaviour (but now seen analytically) as had only previously been observed numerically for local potentials [15-18]. Thirdly, and ultimately perhaps most importantly, we hope later to be able to extend these CLAD sub-approximations to the CCF SUB2 approximations, to include other neglected terms in the SUB2 approach (and perhaps beyond). It will undoubtedly then be necessary to approximate further the integral equations involved for the four-point $S_{2}$-function. In this case the present exact analytic results can be used as a yardstick against which to measure the quality of these further approximations. We note here the analogous precedent for this approach in the work $[6,7]$ on the one-component Coulomb plasma (or "electron gas") using the g.s. CCF. In this case the exact solution for $S_{2}$ in the random-phase approximation was first obtained [6], and later very successfully used [7] in a comparable fashion to give a complete treatment of the electron gas in the metallic density range (where the effective dimensionless coupling constant becomes of the order of unity) using a CCF approach based on a complete SUB2 treatment and beyond. In this case the $\mathrm{CCF}$ treatment gave a microscopic description of the electron gas (which is probably the most well-studied of all quantum many-body systems) which has not been bettered.

We also stress that there are two main aspects of the present calculations on which to focus. The first is the way that the various composite pairs have arisen in this g.s. calculation (and also their role as (de-)excited states which is discussed more fully elsewhere [13]). Concerning this aspect we stress that really for the first time do we have a unified framework in which to imbed and to describe all aspects of pairing (e.g., a possible free bound state and its gradual approach to "dissolution" when immersed in the many-body medium of identical constituent fermions and as the density is increased; the possible appearance for some densities of a second bound state of two quasiparticles of predominantly hole-like character, that arises solely due to the inclusion of hole-hole interactions; the unstable but bound resonance states that exist for densities above which the two previous types of real bound pairs have "dissolved"; Cooper pairs), both for the pairs "in isolation" (i.e., their twobody wavefunctions $\Psi_{2}$ within the medium) and for the system as a whole (which may now condense into a new (de-)excited phase formed of the composites). The second aspect that we stress equally is that quite independently of the existence of any of these various composite-pair states, we have a general solution for the twobody correlations $S_{2}$ of our problem in the physical scattering regime of the twoparticle/two-hole excitations described by $S_{2}$.

Finally, and focussing for the moment just on the first aspect above, we note that looking beyond the present CLAD approximation one may well find that as one includes the effects of extra SUB2 (or higher) terms, that some of these composite pairs are either (i) destroyed by the interactions with these other terms or with higher-order subsystems; or (ii) superseded by more tightly bound higher-order clusters (e.g., in nuclear matter we expect the four-body $\alpha$-particle clustering to be the preferred low-density condensed phase). Alternatively, the composite pairs may turn out to be stable with respect to the inclusion of such other neglected effects (as seems to be the case for Cooper pairing in superconductors) in which case the present 
solutions should provide a good starting-point for a description of the consequent condensed phase built from such composites.

In any case it is of considerable interest to extend the CLAD approximation, and it is our hope that the present work will provide a good starting-point for such extensions. Many authors have considered such extension, generally within different formulations of many-body theory. For example, the extension of either the ppLAD or CLAD approximations to include in the propagation of the intermediate scattering states the effects of interactions with the rest of the system through an averaged one-body potential (and which has the effect of replacing the bare kinetic energies in the left-hand side of Eq. (2.11) of I with the so-called self-consistent hole and/or particle energies), has a long and chequered history. From the ppLAD approach this has led to the well-known Brueckner-Bethe-Goldstone (BBG) approach, in the standard treatment of which, following Brandow [21], the holes are treated self-consistently thus but the particle potential is left at zero. This has the effect in general of introducing a gap at the Fermi surface in the single-particle spectrum, which in turn may easily by itself wipe out the composite pairing that we have observed. An early discussion of this point within the CCF, which only considered particle-particle interactions, was given by Kümmel [22]. More recently similar considerations have been made both within the BBG approach and in the Green's function formulation by the Liège group [23], who discuss the de-merits of introducing such a gap at the Fermi surface and the importance of treating particles and holes symmetrically.

Our own viewpoint is that one should not focus too hard on one single term from the complete SUB2 approach but should rather attempt to incorporate at least approximately all the remaining SUB2 terms. In particular it seems to us to be at least as interesting to investigate the interplay between the short-range correlations (which largely produce the few-body bound clusters focussed on here) and the longrange correlations (which are effective in producing the collective behaviour and, in particular, the "giant resonances" or collective excitations); or equivalently in the language of Kohn and Sherrington [24], to investigate the interference between the type I particle-particle and/or hole-hole pairs discussed here and the so-far neglected type II particle-hole pairs. For such discussions it is clearly important that particles and holes be treated symmetrically from the outset, as in the present CLAD discussion. Finally we are also interested in extending the present approach to at least a qualitative enquiry into the analogous various possibilities of bound threeand four-body clusters and the consequent condensed phases associated with them.

Acknowledgements. This work was largely supported by the Science and Engineering Research Council of Great Britain, and in part by the Polish-U.S. Maria Sklodowska-Curie fund. Their support is gratefully acknowledged. One of us (W.P.) would like to thank the Department of Mathematics at UMIST for their warm and generous hospitality during his stay there. W.P. also acknowledges stimulating discussions with Professor J. Dabrowski.

\section{Appendix: Analytic Structure of the CLAD Function Ln K(z)}

\section{A.1 General Comments; Pairs in Vacuo}

In this Appendix we investigate the analytic structure of the function $\operatorname{Ln} K(z)$ where $K(z)$, defined by Eq. (3.12), has been seen to play a key role in our discussion of pairing. It is clear from the definition 
(3.12) that the function $K(z)$ is analytic in the entire complex $z$-plane with the exception of a branch cut along the positive real axis (or, physically, the scattering regime). The function $\operatorname{Ln} K(z)$ therefore has the same analytic structure except for the addition of any extra branches generated by any zeros (or infinities) in $K(z)$. In particular, as we have seen in Sect. 3, a knowledge of the position of the zeros is essential [and see Eq. (3.32)] for the evaluation of the only unknown functions, namely $P(z)$ and $\bar{P}(z)$, that remain for the complete solution of the CLAD equations.

By analogy with the standard theory of two-body scattering in vacuo, the zeros of the $K$-function are a priori likely to be intimately connected with bound states (or composite pairs). Thus, for the free scattering (i.e., in vacuo) of two particles (taken for ease to have antiparallel spins to avoid the trivial complications of antisymmetry) with relative momentum $\hbar \vec{p}^{\prime}$, we have that the Schrödinger equation in the center-of-mass coordinate system is given by

$$
\left(\nabla^{2}+p^{\prime 2}\right) \psi_{\vec{p}^{\prime}}(\vec{r})=\frac{m}{\hbar^{2}} \frac{1}{\Omega} \int d \vec{r}^{\prime}\left\langle\vec{r}|V| \vec{r}^{\prime}\right\rangle \psi_{\vec{p}^{\prime}}\left(\vec{r}^{\prime}\right) .
$$

Using the usual outgoing-wave (scattering) boundary condition for example, and our separable potential (3.3), the Schrödinger equation (A.1) is readily solved to give in the momentum representation

$$
\begin{aligned}
\psi_{\tilde{p}^{\prime}}(\vec{p}) & =\frac{1}{\Omega}(2 \pi)^{3} \delta\left(\vec{p}-\vec{p}^{\prime}\right)+T_{\vec{p} \vec{p}^{\prime}}^{0} \\
T_{\tilde{p} \vec{p}^{\prime}}^{0} & =-\frac{\lambda}{\Omega} \frac{g(p) g^{*}\left(p^{\prime}\right)}{p^{2}-p^{\prime 2}-i \eta} \frac{1}{K_{0}\left(p^{\prime 2}+i \eta\right)}
\end{aligned}
$$

where the function $K_{0}(z)$ is defined by

$$
K_{0}(z) \equiv 1+\lambda \int_{0}^{\infty} \frac{k^{2} d k}{2 \pi^{2}} \frac{|g(k)|^{2}}{k^{2}-z} .
$$

The reduction of our CLAD solution (3.5) to the free solution (A.2) in the limit $k_{F} \rightarrow 0$ is then readily seen. Thus, from Eqs. (3.6) and (3.13) we readily see that in the limit $k_{F} \rightarrow 0$ (where $\bar{Q} \rightarrow 1$ and $Q \rightarrow 0$ ), then $l\left(p^{2}\right) \rightarrow 1$ and $b(z) \rightarrow K^{-1}(z)$. A similar comparison of Eqs. (A.3) and (3.12) shows that $K(z) \rightarrow K_{0}(z)$ as $k_{F} \rightarrow 0$, as required. Furthermore, the two-body Schrödinger equation is similarly trivially solved in vacuo for bound states to show that the eigenvalue condition for free bound pairs of center-of-mass energy $E_{B} \equiv \hbar^{2} \varepsilon_{B} / m$ is

$$
K_{0}\left(\varepsilon_{B}\right)=0
$$

It would thus seem sensible to examine first the function $K_{0}(z)$, which as we have seen is the limiting form of $K(z)$ for $k_{F} \rightarrow 0$. From the definition (A.3) it is easy to see that $\operatorname{Im} K_{0}(z)$ can vanish nowhere in the complex $z$-plane except on the real axis. On the negative real axis it vanishes identically and on the positive real axis (real scattering regime), $\operatorname{Im} K_{0}\left(p_{c}^{2}\right)=0$ only at those values of $p_{c}^{2}$ where $g\left(p_{c}\right)=0$. Thus in the right-half energy plane, $K_{0}$ has zeros only at those real values $z \rightarrow p_{c}^{2}$ where both $g\left(p_{c}\right)=0$ and $\operatorname{Re} K_{0}\left(p_{c}^{2}\right)=0$ simultaneously. This situation generally only arises in practice by deliberate design, and has in fact been discussed by Tabakin [20] as a method of getting both attraction and repulsion into a one-term separable potential, which is otherwise impossible to achieve. We do not discuss this case further since it adds little to our results. With the exception of deliberately designed potentials like those of Tabakin the only possibility of zeros (for example, for such commonly used separable potentials as that of Yamaguchi [19]) is therefore for negative real values of $z$, which hence correspond. to real bound states as in the derivation of Eq. (A.4). In this case, considering $z \rightarrow-e_{B}$, it is trivial to show that

$$
\operatorname{sgn}\left(d \operatorname{Re} K_{0}\left(-e_{B}\right) / d e_{B}\right)=\operatorname{sgn} \lambda, \quad \forall e_{B}>0
$$



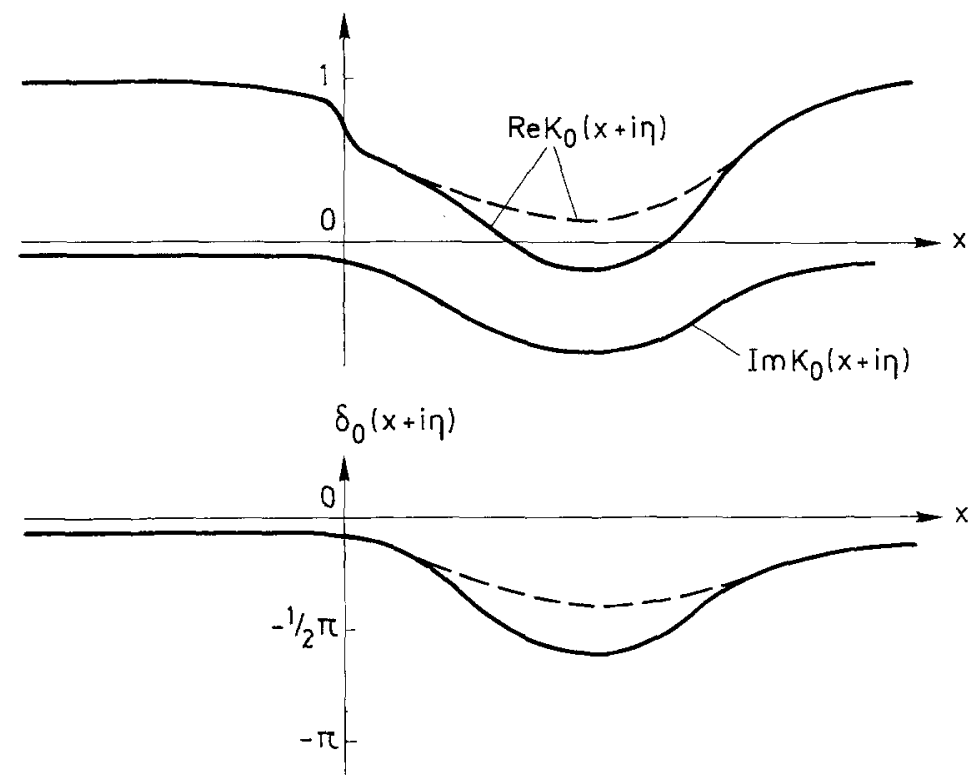

Fig. 1. The real and imaginary parts of the function $K_{0}(z)$ just above the real axis, and the corresponding branch of the phase angle $\delta_{0}$ for which $\delta_{0}$ vanishes at infinity, for an attractive one-term separable potential that supports no free bound state. Two different possibilities are shown for $\operatorname{Re} K_{0}$ and the respective cases for $\delta_{0}$ are shown correspondingly
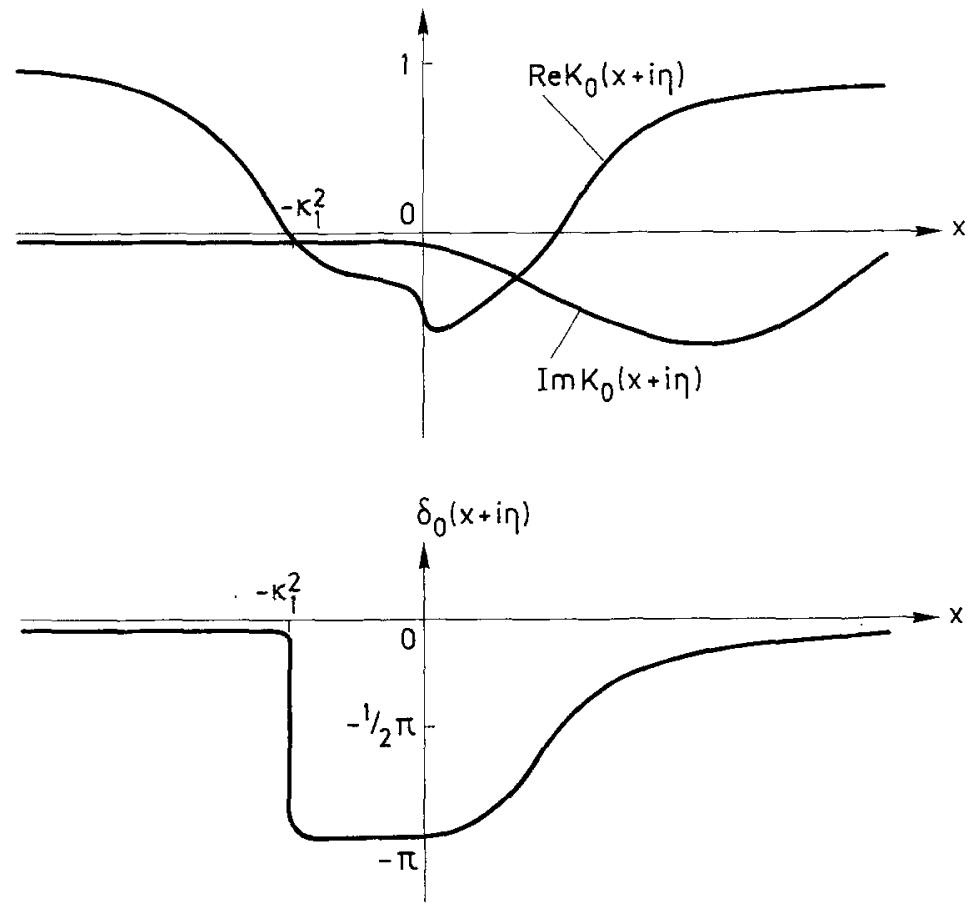

Fig. 2. As in Fig. 1 but for an attractive one-term separable potential that supports a free bound state of energy $E_{B} \equiv-\hbar^{2} \kappa_{1}^{2} / m$ 
Hence, since $K_{0}\left(-e_{B}\right) \rightarrow 1$ as $e_{B} \rightarrow \infty$, these one-term separable potentials must either have no real negative-energy bound states in the case $\lambda>0$ ("repulsive" case), or only the two possibilities of zero or one bound state in the case $\lambda<0$ ("attractive" case) depending on the strength $|\lambda|$ of the attraction.

For purposes of later comparison it is instructive to consider typical behaviour in these various cases of the real and imaginary parts of $K_{0}(z)$ along the real axis, and of the corresponding phase angle $\delta_{0}(z)$ [defined as usual by $\left.K_{0}(z)=\left|K_{0}(z)\right| \exp (i \delta(z))\right]$, chosen again to be the branch on which $\delta_{0}$ vanishes at infinity. Since the repulsive case is of less interest here, we show in Fig. 1 the attractive case $(\lambda<0)$ where a free bound state does not exist, and the corresponding case in Fig. 2 where it does exist at $\varepsilon_{B}=-\kappa_{1}^{2}$. For clarity, we illustrate only the case where the form factor $g(p)$ has no zeros for real positive values of $p$ (as previously discussed). For the moment we note only from Fig. 2 the jump in $\delta_{0}$ of $\pi$ at the point $-\kappa_{1}^{2}$ which is a zero in the function $K_{0}(z)$, or equivalently a branch point of $\operatorname{Ln} K_{0}(z)$.

\section{A.2 Zeros in $K(z)$; General Remarks}

Turning now to the zeros of the CLAD function $K(z)$, we shall first consider by analogy with the free bound states, the real negative zeros. As we shall see, a second type of "bound state", impossible in vacuo and also totally absent in the BG approach to the ladder sum, may appear in certain circumstances. As has previously been discussed [15-17], this new "bound state" may be interpreted as having a strong hole-hole component.

Clearly, the precise structure of the zeros depends on the details of the form factor, the sign and size of the strength parameter $\lambda$, the density of the medium, and now also the total momentum $P$. We first consider below typical behaviour of how the positions of the real zeros vary with $k_{F}$, and then use this information to plot analogous curves to Figs. 1 and 2 for the function $K(z)$.

The function $K(z)$ is given by Eq. (3.12) as

$$
K(z)=1+\lambda \int_{0}^{\infty} \frac{k^{2} d k}{2 \pi^{2}} \frac{|g(k)|^{2}}{k^{2}-z}[\bar{q}(k, P)-q(k, P)] .
$$

Writing $z=x+i y$, where $x$ and $y$ are real, we may decompose $K(z)$ into its real and imaginary parts:

$$
\begin{aligned}
& \operatorname{Re} K(x+i y)=1+\lambda \int_{0}^{\infty} \frac{k^{2} d k}{2 \pi^{2}} \frac{|g(k)|^{2}\left(k^{2}-x\right)}{\left(k^{2}-x\right)^{2}+y^{2}}[\bar{q}(k, P)-q(k, P)], \\
& \operatorname{Im} K(x+i y)=\lambda y \int_{0}^{\infty} \frac{k^{2} d k}{2 \pi^{2}} \frac{|g(k)|^{2}}{\left(k^{2}-x\right)^{2}+y^{2}}[\bar{q}(k, P)-q(k, P)] .
\end{aligned}
$$

It is again clear that $\operatorname{Im} K$ is zero on the negative real axis. However, due to the appearance in the integrand of the non-definite function $(\vec{q}-q)$ which changes sign in the integration region at $k=\left(k_{F}^{2}-\frac{1}{4} P^{2}\right)^{1 / 2} \equiv x_{0}$, for $P<2 k_{F}$, it is now quite possible for $\operatorname{Im} K$ also to be zero elsewhere in the complex plane. Thus the discussion of the zeros of $K(z)$ is considerably more complex than either the free two-body case for $K_{0}(z)$ or the BG case obtained formally from the CLAD case by setting $q(k, P) \rightarrow 0$, as seen from Eq. (3.43).

Restricting ourselves first to the possible zeros at $z=-e_{B}$ on the negative real axis, as indicated above, and considering also just the case $P=0$ for ease, it is apparent that $e_{B}=e_{B}\left(k_{F}\right)$ is the solution to

$$
1+\lambda\left[\int_{k_{F}}^{\infty}-\int_{0}^{k_{F}}\right] \frac{k^{2} d k}{2 \pi^{2}} \frac{|g(k)|^{2}}{k^{2}+e_{B}} \equiv K\left(-e_{B} ; k_{F}\right)=0 .
$$

From Eq. (A.8) we see that for either sign of $\lambda$ there is a possibility of such a zero (for some values of $k_{F}$ ) since one or other of the two integral terms will be negative. The corresponding $K$-function for the BG approach is obtained by neglecting the integral over the range $\left(0, k_{F}\right)$ in Eq. (A.8), and in this case "bound state" zeros are possible only for $\lambda<0$. 
We begin by looking at the case $\lambda<0$. Firstly, if $\lambda$ is not sufficiently negative to support a bound state in vacuo, there remain no possibilities of zeros for $k_{F} \neq 0$. We need consider therefore only the case where a free bound state does exist (at $k_{F}=0$ ). In this case it is then easy to show from Eq. (A.8) that the slope of the curve $e_{B}\left(k_{F}\right)$ is given by

$$
\frac{d e_{B}}{d k_{F}}=\frac{2 k_{F}^{2}\left|g\left(k_{F}\right)\right|^{2}}{k_{F}^{2}+e_{B}}\left[\left(\int_{0}^{k_{F}}-\int_{k_{F}}^{\infty}\right) k^{2} d k \frac{|g(k)|^{2}}{\left(k^{2}+e_{B}\right)^{2}}\right]^{-1} .
$$

Eq. (A.9) then immediately gives that, so long as $g(k) \rightarrow$ const. as $k \rightarrow 0$ (as should be true for all reasonable form factors),

$$
\begin{aligned}
& d e_{B} / d k_{F} \rightarrow 0^{-} \quad \text { as } \quad k_{F} \rightarrow 0, \\
& d e_{B} / d k_{F} \rightarrow 0^{+} \quad \text { as } \quad e_{B} \rightarrow 0,
\end{aligned}
$$

and more generally therefore that the curve $e_{B}\left(k_{F}\right)$ must "backbend" as in Fig. 3, in contrast to the BG case where the slope is everywhere negative. It is clear without a detailed derivation by appealing to continuity in $P$ that this same behaviour must also persist for small enough non-zero values of $P$. (We also note that in the CLAD case, although we have shown the slope of the $e_{B}\left(k_{F}\right)$ curve becoming infinite once, the proof presented only rigorously shows that one must have an odd number of such infinities. In the ensuing discussion we restrict ourselves to the simplest case shown in Fig. 3.)

We see that in the CLAD approximation for separable potentials sufficiently attractive to bind a two-body state in vacuo, for small enough values of $P$ at least the density regime divides into three regions. (For large enough values of $P$ these "bound-state" zeros will all finally disappear.) In region $I$, $0<k_{F}<k_{F m}$, there is just one zero (or "bound pair"). In region II, $k_{F m}<k_{F}<k_{F M}$, a second "bound state" appears. This, represented by the lower limb of the curve, can only be interpreted as a predominantly hole-hole-like pair. As $k_{F}$ approaches the upper critical value $k_{F M}$ from below the two zeros approach each other and then coincide at $k_{F M}$. In region III, $k_{F}>k_{F M}$, no such real zeros are possible. However, it would be most surprising if the "bound states" completely "dissolved" in this region. What actually must happen is clear without going into details. Thus the two zeros coincide at $k_{F} \rightarrow k_{F M}$ and then for $k_{F}>k_{F M}$, they move into the complex plane as conjugate "resonantantiresonant" pairs. Thus in region III we find complex zeros corresponding to the formation of resonant states with finite lifetimes. How close these states remain to the real axis and their ultimate fate for very large values of $k_{F}$ (i.e., whether they move into the right half plane), depends on the details of the interaction. We intend to discuss these resonant states in more detail in a later paper. We note also that the critical values $k_{F m}$ and $k_{F M}$ themselves depend on the total momentum $P$. For given density there is a critical value of $P$ at which $k_{F m}$ and $k_{F M}$ tend to zero, and above which no real "bound states" exist.

In the case of repulsive potentials $(\lambda>0)$, the BG approximation clearly does not permit bound state zeros. However, it is clear that if the inverse of the original repulsive potential (i.e., changing only

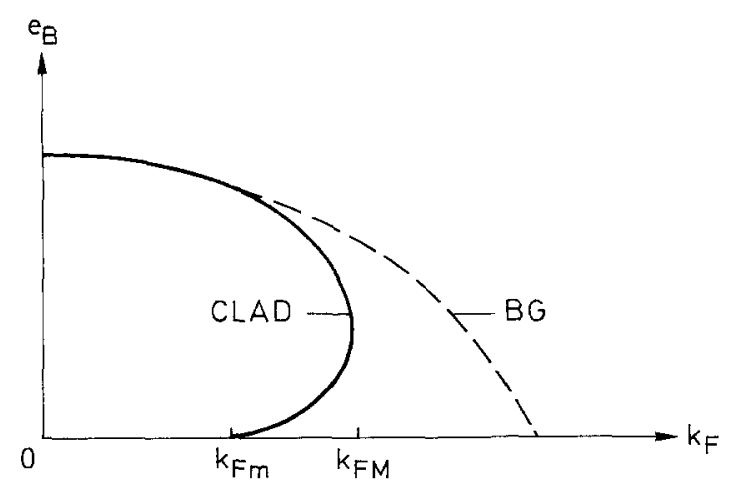

Fig. 3. Schematic plot of the real negative zeros at $z=-e_{B}$ (for small enough values of total momentum $P$ ) in the CLAD function $K(z)$ and its analogue for the BG case, as a function of Fermi wavenumber $k_{F}$, for the case of an attractive one-term separable potential that supports a free bound state 
Fig. 4. As in Fig. 3 (for the CLAD case) but for a repulsive one-term separable potential whose inverse (i.e., $\lambda \rightarrow-\lambda$ ) supports a free bound state

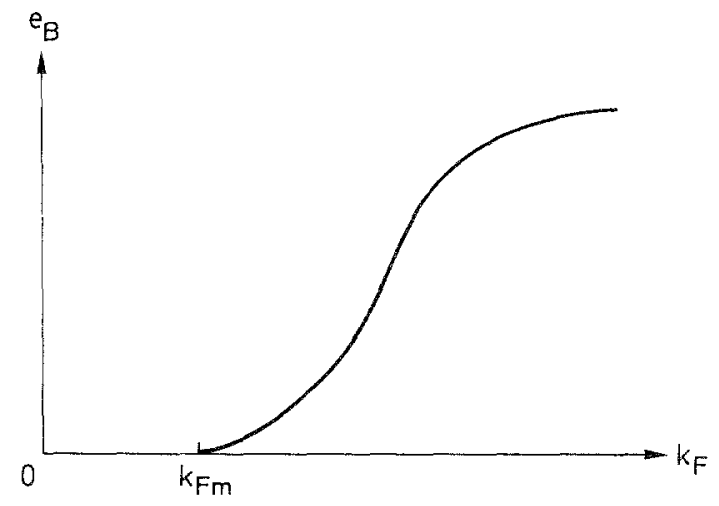

$\lambda \rightarrow-\lambda$ ) could support a free bound state, then for large enough values of $k_{F}$ there must again be a real zero possible in the CLAD $K$-function. An equivalent analysis of the derivatives as in the attractive case allows us to make a similar schematic plot in Fig. 4 for the present "sufficiently repulsive" case as Fig. 3 was for the "sufficiently attractive" case. (Again we have sketched the $e_{B}\left(k_{F}\right)$ curve as if it were monotonic increasing, although this is not guaranteed.)

\section{A.3 $\operatorname{Re} K(x+i \eta), \operatorname{Im} K(x+i \eta)$ and Phase $\delta(x+i \eta)$}

We now use the above results to give sketches for some of the various CLAD possibilities analogous to Figs. 1 and 2 for the free pair case. Before doing so, however, we shall need some further properties of the $K$-function. First, from Eq. (A.5) we have for real $p$

$$
\operatorname{Im} K\left(p^{2}+i \eta\right)=\left\{\begin{aligned}
(4 \pi)^{-1} \lambda|p \| g(p)|^{2} \bar{q}(p, P), & p^{2}>x_{0}^{2}, \\
-(4 \pi)^{-1} \lambda|p \| g(p)|^{2} q(p, P), & p^{2}<x_{0}^{2},
\end{aligned}\right.
$$

and hence the sign of $\operatorname{Im} K(z)$ can be definitely decided everywhere in the physical scattering regime (i.e., just above or just below the positive real axis). Conversely, close to the negative real axis, where $\operatorname{Im} K(z)$ approaches zero, we can decide on the sign of the (infinitesimal) $\operatorname{Im} K(-e+i \eta)$ in terms of the slope of $\operatorname{Re} K(-e+i \eta)$ from the relation

$$
\operatorname{Im} K(-e+i \eta)=\eta d \operatorname{Re} K(-e+i \eta) / d(-e), \quad e>0,
$$

which is readily proved from Eqs. (A.6) and (A.7).

Secondly we prove an important result about the sign of $\operatorname{Re} K\left(x_{0}^{2}+i \eta\right)$ in the case that $K(z)$ has at least one zero in the left-half complex plane. Thus if there exist real $e>0$ and $\Gamma$ such that $K(-e+i \Gamma)=0$, we may write from Eq. (A.5)

$$
1=-\lambda \int_{0}^{\infty} \frac{k^{2} d k}{2 \pi^{2}} \frac{|g(k)|^{2}}{k^{2}+e-i \Gamma}[\bar{q}(k, P)-q(k, P)] .
$$

Substituting into Eq. (A.5) from the real part of the above result gives the relation

$$
\begin{aligned}
\operatorname{Re} K\left(x_{0}^{2}+i \eta\right) & =\lambda \int_{0}^{\infty} \frac{k^{2} d k}{2 \pi^{2}}|g(k)|^{2}\left(\frac{1}{k^{2}-x_{0}^{2}}-\frac{k^{2}+e}{\left(k^{2}+e\right)^{2}+\Gamma^{2}}\right)[\bar{q}(k, P)-q(k, P)] \\
& =\lambda \int_{0}^{\infty} \frac{k^{2} d k}{2 \pi^{2}}|g(k)|^{2} \frac{\left(k^{2}+e\right)\left(e+x_{0}^{2}\right)+\Gamma^{2}}{\left(k^{2}-x_{0}^{2}\right)\left[\left(k^{2}+e\right)^{2}+\Gamma^{2}\right]}[\bar{q}(k, P)-q(k, P)] .
\end{aligned}
$$

In the above form we note that the integrand is everywhere positive since $[\bar{q}(k, P)-q(k, P)]$ is negative (positive) for $0<k^{2}<x_{0}^{2}\left(k^{2}>x_{0}^{2}\right)$, respectively. We thus have the important result 


$$
\lambda \operatorname{Re} K\left(x_{0}^{2}+i \eta\right)>0
$$

in case there exist real positive $e$ and real $\Gamma$ such that $K(-e+i \Gamma)=0$.

Finally we note that apart from zeros in the function $K(z)$ we should also consider any infinities since these will also be branch points of $\operatorname{Ln} K(z)$. However, it is clear from Eq. (A.5) that such singularities can arise only for $z$ at points on the positive real axis where the integrand itself is discontinuous. By inspection we see that this occurs only for the case $P=0$ at the Fermi surface $z \rightarrow k_{F}^{2}$, and in this case

$$
\operatorname{Re} K\left(p^{2}+i \eta\right) \underset{p \rightarrow k_{F}}{\longrightarrow}-\frac{\lambda}{2 \pi^{2}} k_{F}\left|g\left(k_{F}\right)\right|^{2} \ln \left|p^{2}-k_{F}^{2}\right|, \quad P=0 .
$$

For non-zero total momentum $P$ there are no such singularities anywhere in $K(z)$. The singularity (A.14) is well known (see, e.g., ref. [25]) to lead in the case $\lambda<0$ to the phenomenon of Cooper pairing, and we therefore do not discuss this further here, preferring to return to this point in a later paper.

We are now in a position to discuss in turn the following cases (for $\lambda<0$ ):

I. Strong attraction (i.e., sufficient to bind a pair in vacuo); with $0<k_{F}<k_{F m}$, as in Fig. 3.
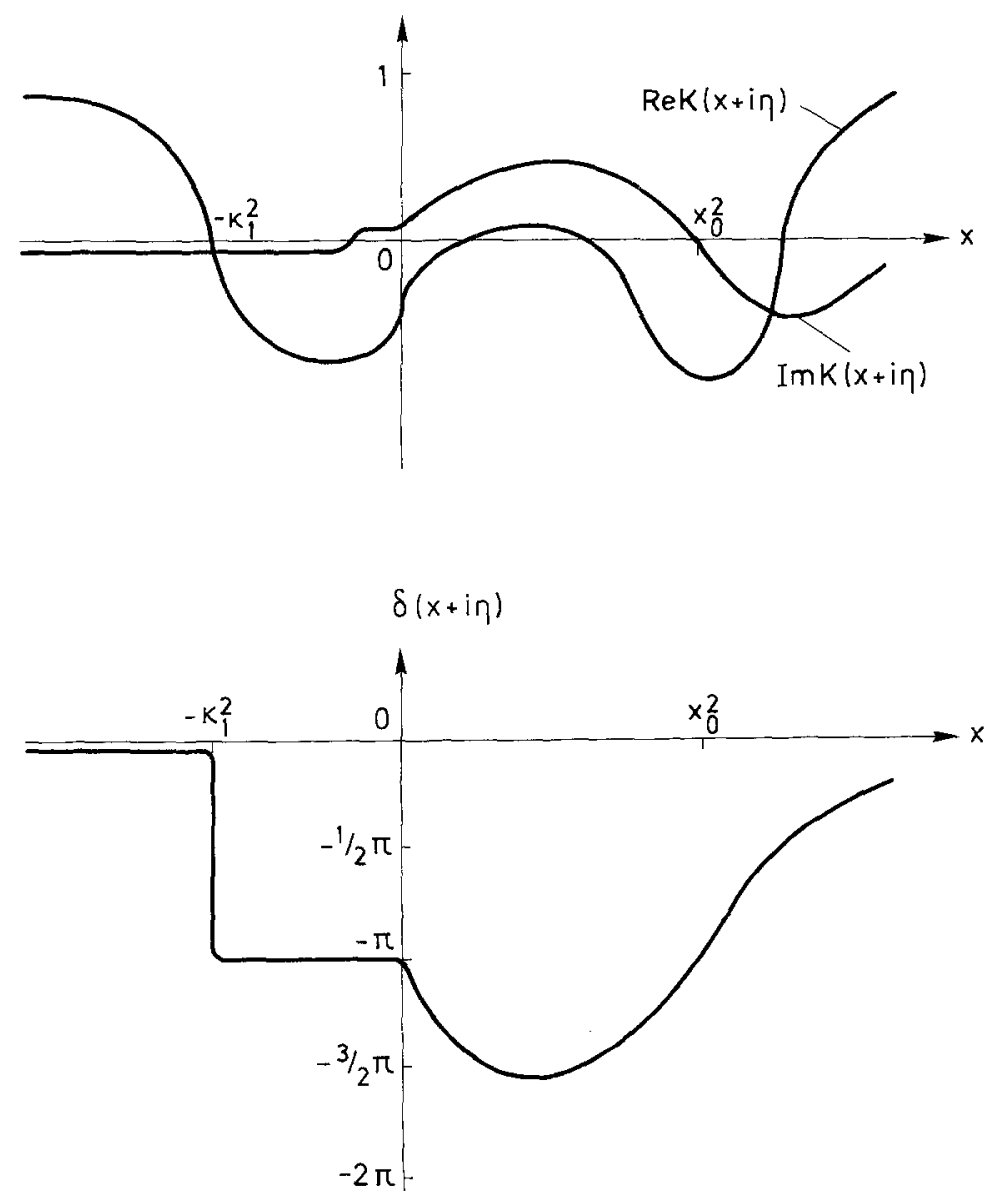

Fig. 5. The real and imaginary parts of the CLAD function $K(z)$ just above the real axis and the corresponding branch of the phase angle $\delta$ which becomes zero at infinity, for an attractive one-term separable potential that supports a free bound state. Shown is the case $0<k_{F}<k_{F_{m}}$ where one "bound pair" exists at $z=-\kappa_{1}^{2}$ 
Exact Model Ground-State Results for Generalised Ladders
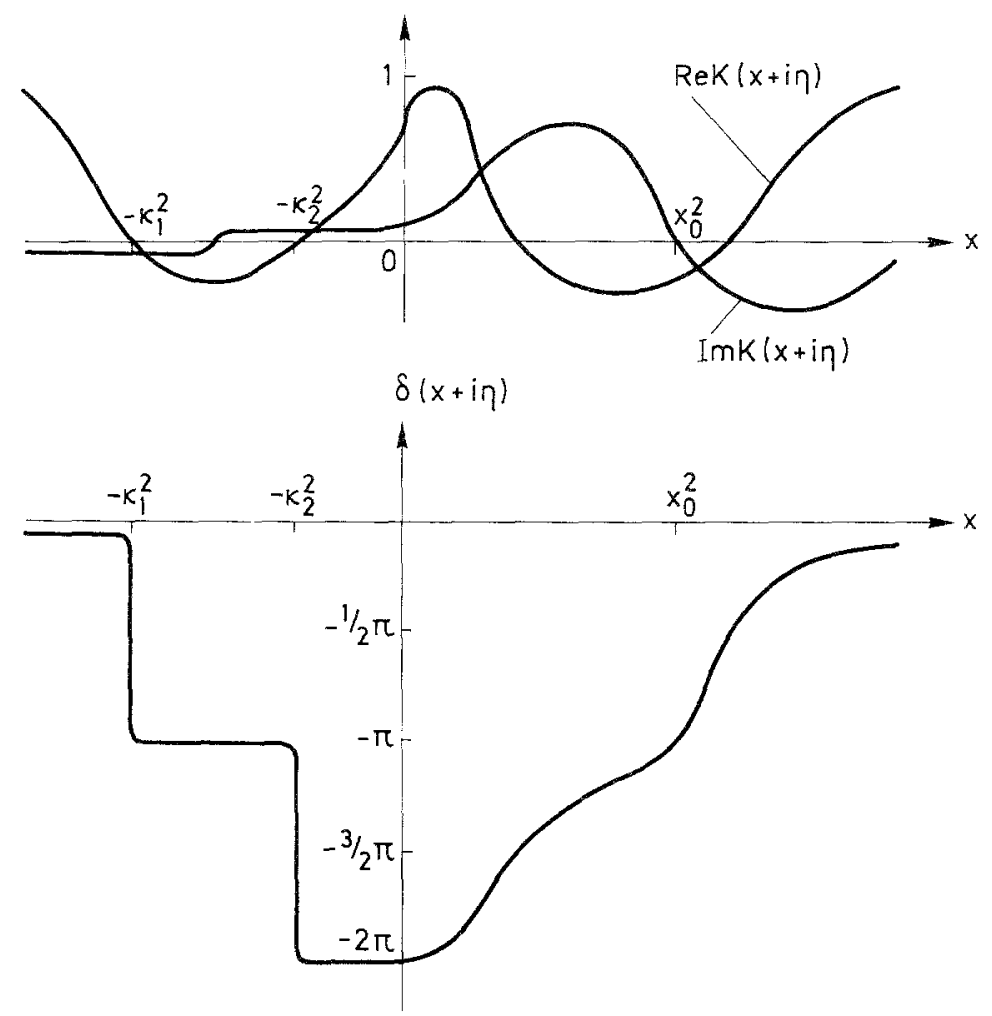

Fig. 6. As in Fig. 5, but for the case $k_{F m}<k_{F}<k_{F M}$ where two "bound pairs" exist at $z=-\kappa_{1}^{2}$ and $z=-\kappa_{2}^{2}$
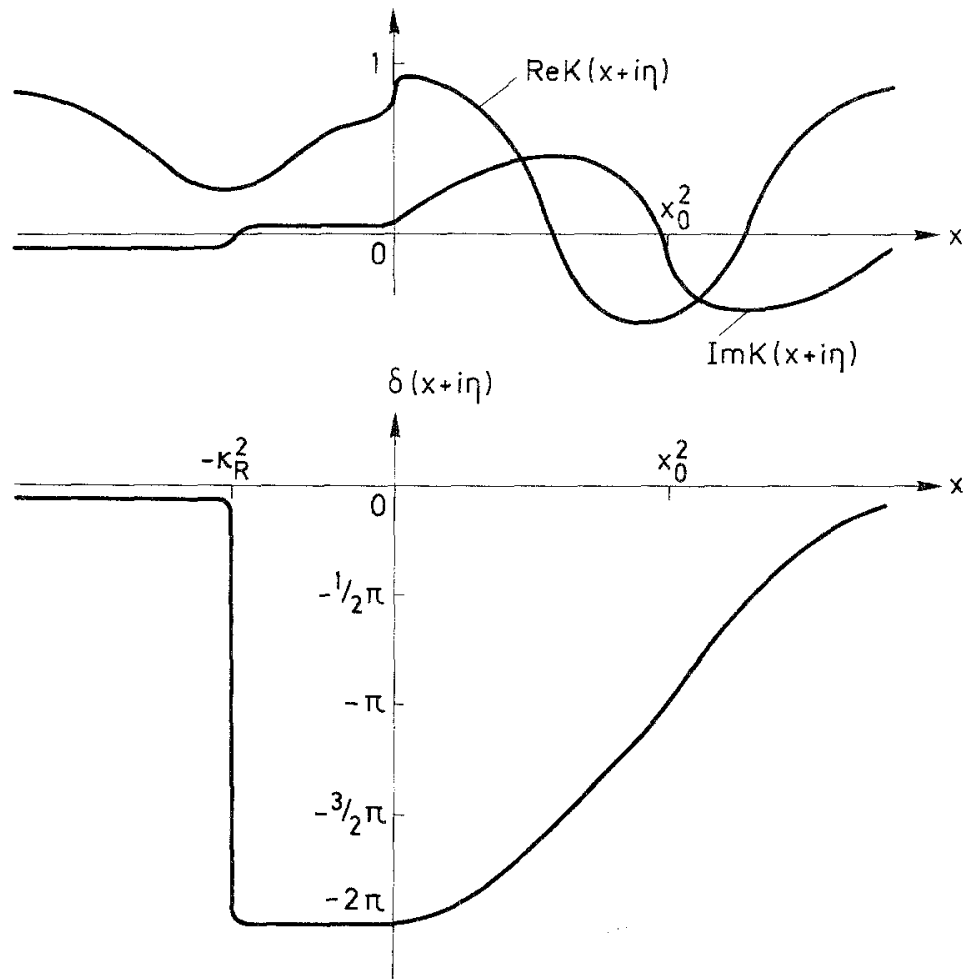

Fig. 7. As in Fig. 5, but for the case $k_{F}>k_{F M}$ and where two "bound resonance pairs" exist at $z=-\kappa_{R}^{2} \pm i \Gamma_{R}$ 
II. Strong attraction; with $k_{F m}<k_{F}<k_{F M}$.

III. Strong attraction; with $k_{F}>k_{F M}$ and a pair of complex resonance zeros in $K(z)$ at $z=$ $-e_{R} \pm i \Gamma_{R}, e_{R}>0$.

IV. Weak attraction (i.e., insufficient to bind a pair in vacuo).

Case I. In this case we simply have one zero at $z=-\kappa_{1}^{2}$ on the negative real axis, and Eqs. (A.11)-(A.13) enable us to plot the phase angle in Fig. 5, which is analogous to the case in vacuo shown in Fig. 2.

Case II. In this region we now have two zeros, namely at $z=-\kappa_{1}^{2}$ and $-\kappa_{2}^{2}$ on the negative real axis, and similar considerations allow us to plot the phase angle in Fig. 6. We see in this case the separate jumps of $\pi$ in the phase angle at both zeros.

Case III. In this region the above two real poles have moved into the complex plane to positions $z=-\kappa_{R}^{2} \pm i \Gamma_{R}$. The function $\operatorname{Ln} K(z)$ thus has a branch cut joining these, and if we choose the cut simply to be the straight line joining them, then the phase angle now appears as in Fig. 7, where the jump of $2 \pi$ now reflects that we should indent around this branch in the usual way for the phase to return to zero along the positive axis, i.e. to ensure that we stay on our chosen branch of $\delta(z)$ which becomes zero at infinity.

Case IV. This case can be handled similarly to the above cases, but since it is of less direct interest, we shall not consider it further.

The analogous possibilities in the case of repulsive potentials $(\lambda>0)$ may also be handled similarly.

\section{References}

1. Bishop, R. F., Piechocki, W., Stevens, G. A.: Few-Body Systems 4, 161 (1988)

2. Bethe, H. A., Goldstone, J.: Proc. R. Soc. Lond. A238, 551 (1957)

3. Chisholm, J. S. R., Squires, E. J.: Nucl. Phys. 13, 156 (1959)

4. Mehta, M. L.: Nucl. Phys. 12, 333 (1959)

5. Salpeter, E. E., Bethe, H. A.: Phys. Rev. 84, 1232 (1951)

6. Bishop, R. F., Lührmann, K. H.: Phys. Rev. B17, 3757 (1978)

7. Bishop, R. F., Lührmann, K. H.: Phys. Rev. B26, 5523 (1982)

8. Bohm, D., Pines, D.: Phys. Rev. 92, 609 (1953)

9. Muskhelishvili, N. I.: Singular Integral Equations. Groningen: Noordhoff 1953

10. Omnès, R.: Nuovo Cim. 8, 316 (1958)

11. Emrich, K.: Nucl. Phys. A351, 379 (1981)

12. Emrich, K.: Nucl. Phys. A351, 397 (1981)

13. Bishop, R. F., Piechocki, W., Stevens, G. A.: to be published

14. Kümmel, H.: Nucl. Phys. A317, 199 (1979)

15. Bishop, R. F., Strayer, M. R., Irvine, J. M.: Phys. Rev. A10, 2423 (1974)

16. Bishop, R. F., Strayer, M. R., Irvine, J. M.: J. Low Temp. Phys. 20, 573 (1975)

17. Ghassib, H. B., Bishop, R. F., Strayer, M. R.: J. Low Temp. Phys. 23, 393 (1976)

18. Bishop, R. F., Ghassib, H. B., Strayer, M. R.: Phys. Rev. A13, 1570 (1976)

19. Yamaguchi, Y.: Phys. Rev. 95, 1628 (1954)

20. Tabakin, F.: Phys. Rev. 174, 1208 (1968)

21. Brandow, B. H.: Phys. Rev. 152, 863 (1966); Rev. Mod. Phys. 39, 721 (1967)

22. Kümmel, H.: Nucl. Phys. 22, 177 (1961)

23. Jeukenne, J.-P., Lejeune, A., Mahaux, C.: Nucl. Phys. A245, 411 (1975); Mahaux, C.: in: Nuclear Physics (Dasso, C. H., Broglia, R. A., Winther, A., eds.), p. 319. Amsterdam: North-Holland 1982 
24. Kohn, W., Sherrington, D.: Rev. Mod. Phys. 42, 1 (1970)

25. Fetter, A. L., Walecka, J. D.: Quantum Theory of Many-Particle Systems, p. 320. New York: McGraw-Hill 1971

Received March 7, 1988; accepted for publication April 12, 1988 